\title{
From Service Member to Student: Predictors of Psychological Help-Seeking Attitudes in Student Servicemembers and Veterans
}

\author{
Melissa A Noble
}

Follow this and additional works at: https://researchrepository.wvu.edu/etd

\section{Recommended Citation}

Noble, Melissa A, "From Service Member to Student: Predictors of Psychological Help-Seeking Attitudes in Student Servicemembers and Veterans" (2018). Graduate Theses, Dissertations, and Problem Reports. 7221.

https://researchrepository.wvu.edu/etd/7221

This Dissertation is protected by copyright and/or related rights. It has been brought to you by the The Research Repository @WVU with permission from the rights-holder(s). You are free to use this Dissertation in any way that is permitted by the copyright and related rights legislation that applies to your use. For other uses you must obtain permission from the rights-holder(s) directly, unless additional rights are indicated by a Creative Commons license in the record and/ or on the work itself. This Dissertation has been accepted for inclusion in WVU Graduate Theses, Dissertations, and Problem Reports collection by an authorized administrator of The Research Repository @ WVU.

For more information, please contact researchrepository@mail.wvu.edu. 
From Service Member to Student: Predictors of Psychological Help-Seeking Attitudes in Student Servicemembers and Veterans

Melissa A. Noble, M.Ed.

Dissertation submitted to the College of Education and Human Services at West Virginia University

in partial fulfillment of the requirements for the degree of

Doctor of Philosophy in

Counseling Psychology

Monica Leppma, Ph.D., Chair

G. David Allen, Ph.D.

Wade G. Livingston, Ph.D.

Lisa F. Platt, Ph.D.

Christine Schimmel, Ed.D.

Department of Counseling, Rehabilitation Counseling, and Counseling Psychology

Morgantown, West Virginia

2018

Keywords: Student servicemembers, mental health stigma, psychological help-seeking Copyright 2018 Melissa A. Noble 


\section{ABSTRACT \\ From Service Member to Student: Predictors of Psychological Help-Seeking Attitudes in Student Servicemembers and Veterans}

\section{Melissa A. Noble, M.Ed.}

Post-secondary institutions have experienced increased enrollment of student servicemembers and veterans (SSM/V), thus prompting renewed interest in the academic and acculturation needs of SSM/V. Despite the breadth of psychological literature surrounding military and college student populations, there is a gap in knowledge concerning the psychological needs of SSM/V. The primary aim of this study was to explore predictors of attitudes toward psychological helpseeking among SSM/V. In the spring of 2017, SSM/V $(n=103)$, including 26 females and 77 males, completed self-report questionnaires. Results from the study demonstrated that selfstigma, public-stigma, and gender were significantly correlated with attitudes toward psychological help-seeking. A multiple regression analysis revealed that self-stigma and gender significantly predicted attitudes toward psychological help-seeking. Consistent with previous studies, self-stigma fully mediated the significant relationship between public-stigma and attitudes toward psychological help-seeking. Ultimately, this study highlights the process in which external events adopt internal psychological significance and furthers the discourse surrounding the specific psychological needs of SSM/V. 
Table of Contents

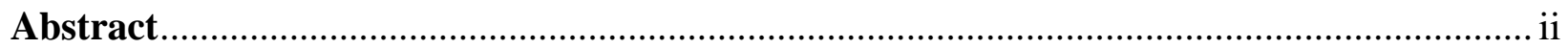

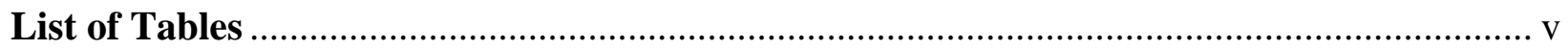

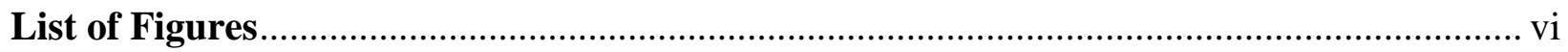

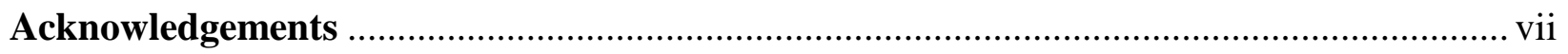

Chapter 1: Introduction and Literature Review.................................................... 1

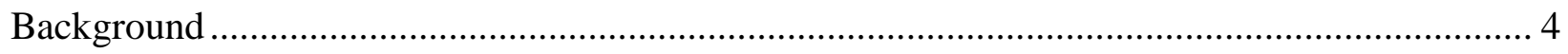

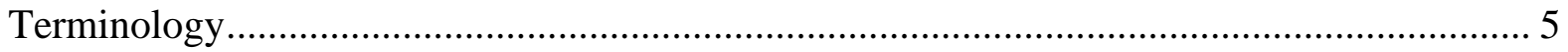

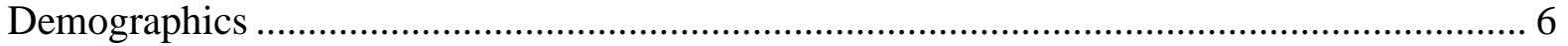

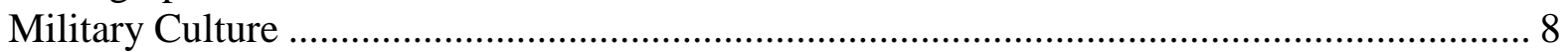

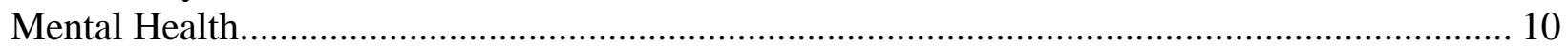

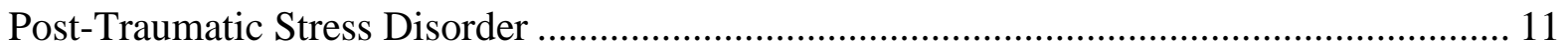

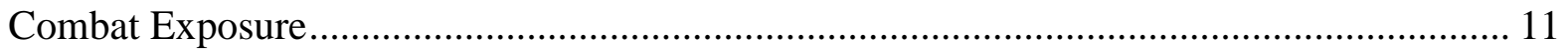

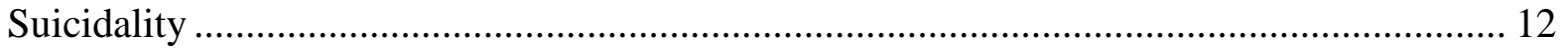

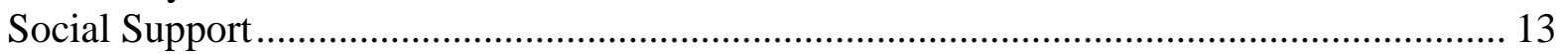

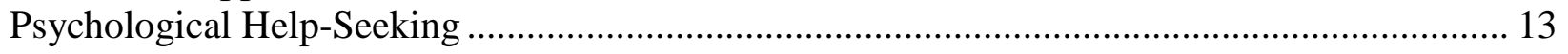

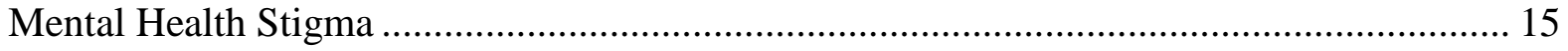

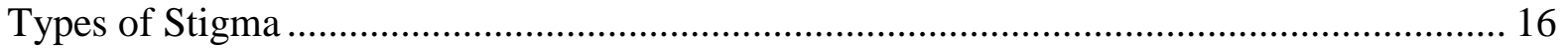

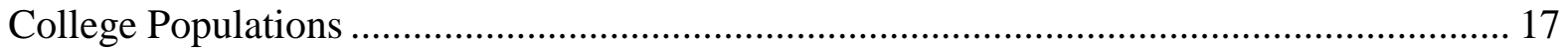

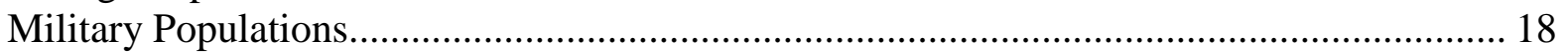

Student Servicemembers and Veterans....................................................................... 20

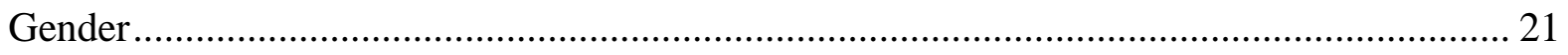

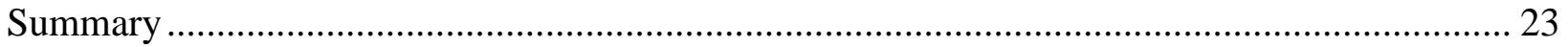

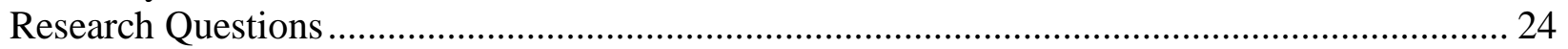

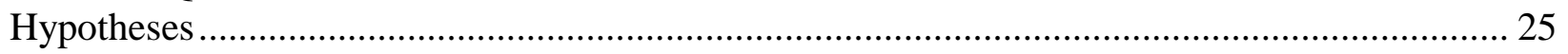

Chapter 2: Research Design and Methodology ......................................................... 26

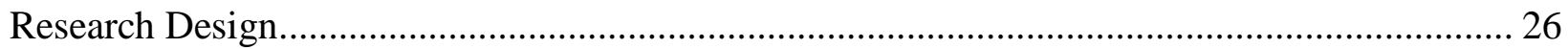

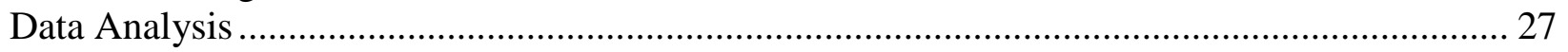

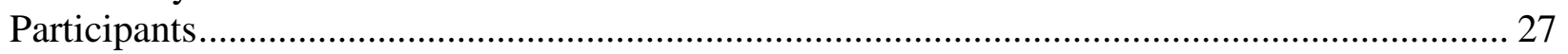

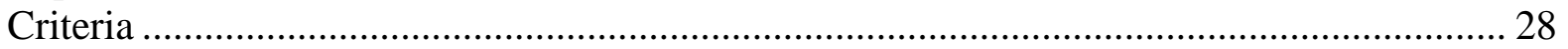

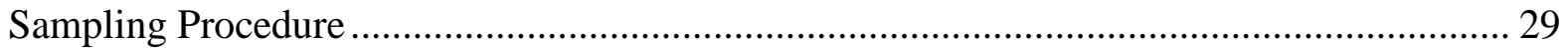

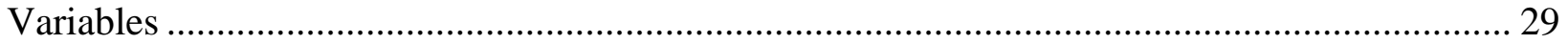

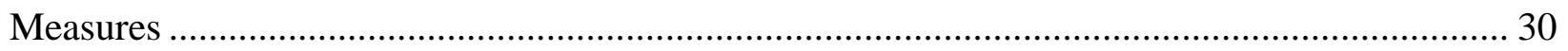

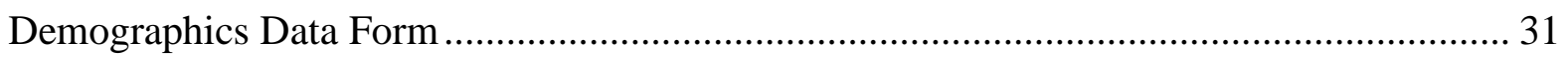

Self-Stigma of Seeking Help Scale ........................................................................... 31

Stigma Scale for Receiving Psychological Help Scale ................................................... 32

Attitudes Toward Seeking Professional Help Scale - Short Form.................................... 33

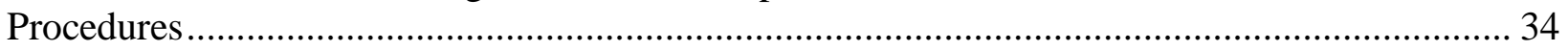




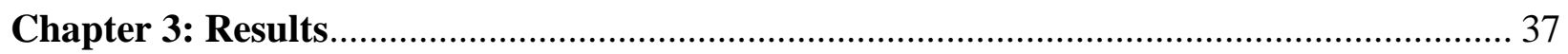

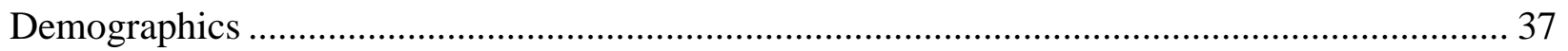

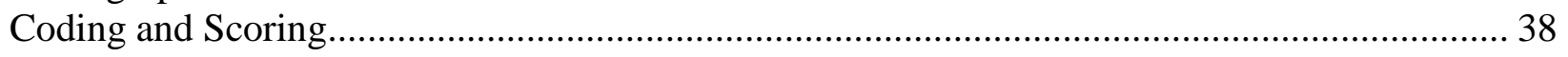

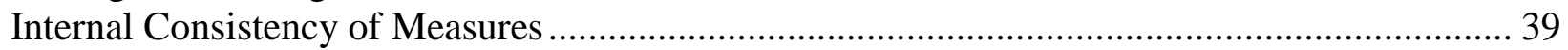

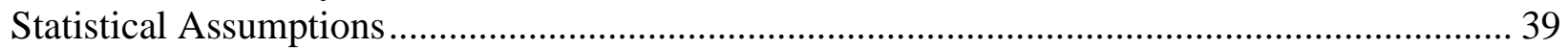

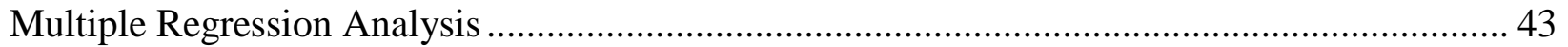

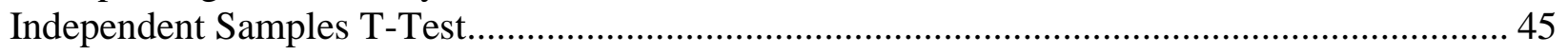

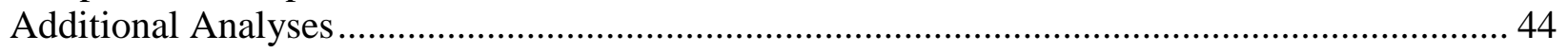

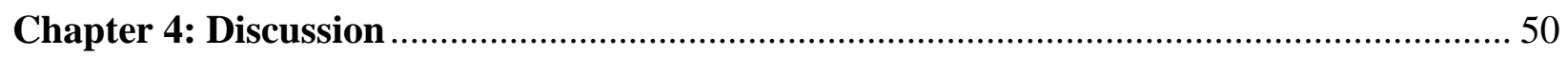

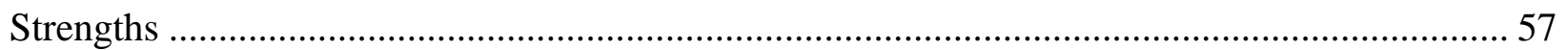

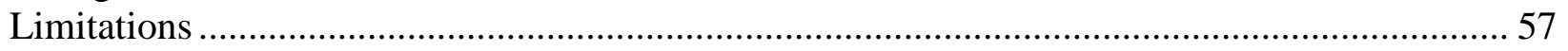

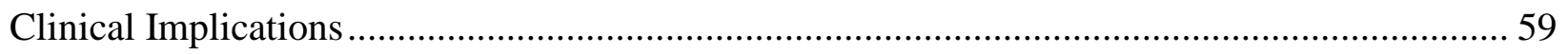

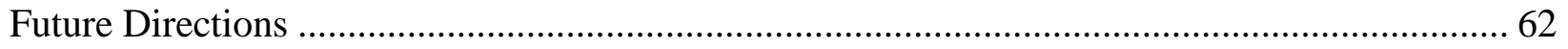

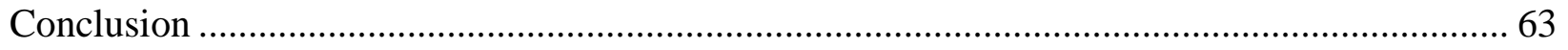

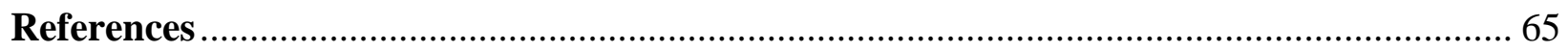

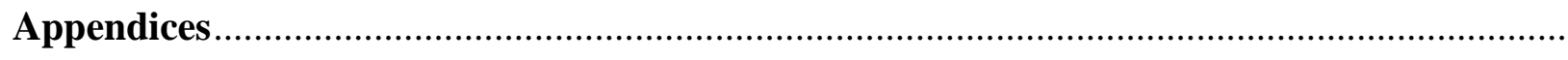

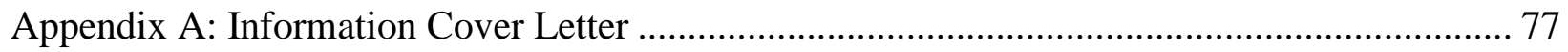

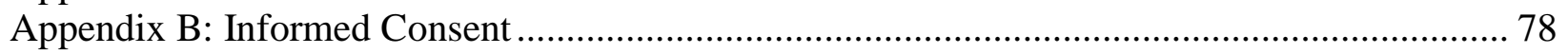

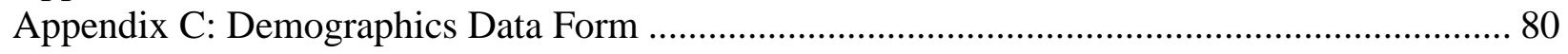

Appendix D: Self-Stigma of Seeking Help Scale ............................................................ 82

Appendix E: Stigma Scale for Receiving Psychological Help Scale ...................................... 84

Appendix F: Attitudes Toward Seeking Professional Help Scale - Short Form........................ 85

Appendix G: Closing Statement and Resources ........................................................... 87 


\section{List of Tables}

Table 1: Demographics by Combat and Military Status...................................................... 38

Table 2: Correlations Among Possible Predictor Variables ................................................... 44

Table 3: Regression Analysis of Predictor Variables ........................................................ 46

Table 4: Independent Samples T-Test of Psychological Help-Seeking Score by Gender........... 46

Table 5: Correlations of Additional Possible Predictor Variables ........................................... 47

Table 6: Self-Stigma as a Mediator of Public-stigma and Help-Seeking ............................... 49 


\section{List of Figures}

Figure 1: Scatterplot of Studenized Residuals and Unstandardized Predicted Values ............... 41

Figure 2: Standardized Residual Histogram of Psychological Help-Seeking Score .................. 41

Figure 3: P-P Plot of Regression Standardized Residual ................................................. 42

Figure 4: Boxplot of Psychological Help-Seeking Score by Gender ..................................... 42

Figure 5: Mediation Model with Public-Stigma, Self-Stigma, and Help-Seeking ..................... 49 


\section{Acknowledgements}

Craig, thank you for your love and support across the miles and years. As my husband and partner-in-crime, you bring joy to even my toughest days. I look forward to our many adventures to come! Mom and Dad, words cannot express how much I appreciate your unwavering support. Collectively, you have shaped me into the person and professional I am today. Thank you for encouraging each of your girls to march to the beat of their own drum. Anita, Kara, and Sami, I am so thankful for sisters like you. You bring so much light to my life and to the world around you, thank you for everything. Foster family, thank you for supporting me and Craig throughout this program. I am incredibly fortunate to have married into such a great family. To my closest friends, I appreciate your relentless support. Our texts, phone dates, and weekend trips have demonstrated that friends truly are the family you choose. To my clinical supervisors, thank you for encouraging my growth as a practitioner. Your influence has made me not only a better practitioner but a better person. To Dr. Leppma and my committee members, thank you for your support and guidance throughout this process. Wade, thank you for inspiring my decision to pursue a doctoral program. Without you, this dissertation would have never happened. Finally, to the brave military servicemen and women, including my family and friends, who have served and continue to serve our country. My research is but a small thank you for the sacrifices you make every day. 


\section{Chapter 1: Introduction and Literature Review}

The landscape of higher education reflects an increasingly diverse and dynamic environment. Unprecedented changes in technology and legislation have broadened access to postsecondary education and have diversified colleges and universities across the United States. For example, institutions have experienced significant increases in military student enrollment (National Conference of State Legislatures, 2014). This growth is primarily attributed to ongoing conflicts in the Middle East and subsequent efforts, such as the Post 9/11 Veterans Educational Assistance Act of 2008 (i.e., Post-9/11 GI Bill), to aid servicemembers in the transition from military service to civilian life. The Post-9/11 GI Bill represents a concerted effort to provide postsecondary benefits to current servicemembers and veterans. Due to these changes, military student enrollment is expected to rise in the coming years, an outcome that has prompted renewed interest in the experiences of student servicemembers and veterans (SSM/V; Barry, Whiteman, \& Wadsworth, 2014).

Considering these anticipated increases in military student enrollment, it is imperative that colleges and universities are prepared to offer targeted support for SSM/V (e.g. Barry et al., 2014; DiRamio, Ackerman, DiRamio, \& Garza Mitchell, 2008; Livingston, Scott, Havice, \& Cawthon, 2012; Rumann \& Hamrick, 2010). Like other nontraditional students, SSM/V experience adjustment and acculturation challenges in higher education (Ackerman, DiRamio, \& Garza Mitchell, 2009; DiRamio et al., 2008; Griffin \& Gilbert, 2015). Ultimately, SSM/V must negotiate two salient identities: military service member and college student. At times, these roles may appear to conflict with one another as the adjustment process is further complicated by cultural differences between the military and academia (Livingston, 2009; Rumann \& Hamrick, 2010). For example, while the military tends to emphasize structure and teamwork, higher 
education promotes independence and autonomy. These inherent differences may pose barriers for SSM/V as they assimilate into university life.

In addition to adjustment and acculturation issues (Livingston, 2009; Rumann \& Hamrick, 2010), SSM/V may experience difficulties related to seeking necessary support. Helpseeking is a term used to describe a person's willingness to engage in assistance from others (Rickwood, Deane, Wilson, \& Ciarrochi, 2005). Existing research has examined help-seeking among SSM/V in terms of academic and financial support. Findings from Livingston (2009), for example, demonstrated a general reluctance among student veterans to secure help. The researcher concluded,

Self-reliance and individual will were both positive and negative examples of self-coping resources. In other words, student veterans' greatest strength could also be their greatest weakness. Participants described an aversion to help and a tendency to rely on individual will to persevere. While the dedication and strength of character are admirable, student veterans' self-reliance limited opportunities for support, especially academic support. (Livingston, 2009, pp. 181-182)

This study sought to expand on these findings by exploring help-seeking in terms of psychological support among SSM/V, a topic that remains largely unexplored in the literature with regards to this population.

Psychological help-seeking is a specific form of help-seeking used to describe a person's willingness to engage in mental health services. In previous studies, this construct has been assessed using psychometric measurements like the Attitudes Toward Seeking Professional Psychological Help Scale (ATSPPH; Fischer \& Turner, 1970). Psychological help-seeking has been widely studied among non-student military members, in addition to college students. 
Results from these empirical investigations have consistently demonstrated that male gender and internalized stigma (i.e. self-stigma) are negatively associated with psychological help-seeking (Bonar, Bohnert, Walters, Ganoczy, \& Valenstein, 2014; Held \& Owens, 2012; Topkaya, 2014). As discussed, SSM/V represent a unique group of individuals as they encompass both the military and college student populations. Considering that previous studies have focused on psychological help-seeking among military members and college students, it is fitting that future studies would examine this construct among SSM/V. Ultimately, this line of research could provide valuable information on variables that impact mental health treatment, especially within the context of university counseling.

Despite a gap in knowledge concerning psychological help-seeking among SSM/V, previous literature has demonstrated unmet mental health needs within this population (Bonar et al., 2014). For example, Barry et al. (2014) found a significant relationship between SSM/V status and psychological symptomatology, particularly among those individuals exposed to combat. The literature has cited several specific psychological concerns among combat-exposed veterans including elevated levels of generalized anxiety, depression, post-traumatic stress disorder (PTSD), high-risk alcohol use, and suicidality (Bonar et al., 2014; Rudd, Goulding, \& Bryan, 2011). Extant literature has also demonstrated no significant differences in terms of symptomology between SSM/V and non-student servicemembers and veterans (Bonar et al., 2014). In general, there is a high prevalence of mental health concerns but a low rate of treatment utilization among both of these populations, especially among military students (Barry et al., 2014).

The expected rise in military enrollment and lack of knowledge concerning psychological help-seeking in SSM/V provided the basis for this study. Building on previous investigations 
concerning military servicemembers and university students, this study examined predictors of psychological help-seeking, namely gender and stigma, among SSM/V. Results from this study could be used to inform best practices in higher education, including college student counseling. Ultimately, this study is intended to expand the knowledgebase concerning SSM/V and to advance the science, practice, and scholarly discourse surrounding military psychology and college student counseling. The remainder of this chapter will provide an overview of the relevant literature and a summary of this study, research questions, and hypotheses.

\section{Literature Review}

\section{Background}

The United States government has a long history of providing educational assistance to servicemembers, beginning with the Servicemen's Readjustment Act of 1944, or GI Bill, following World War II. Most recently, the Post-9/11 GI Bill was passed in response to ongoing conflicts in Afghanistan and Iraq such as Operation Enduring Freedom (OEF) and Operation Iraqi Freedom (OIF). This legislation provides educational benefits for individuals who participated in active duty service after September 11, 2001 including tuition and housing assistance and a monthly stipend for eligible SSM/V and dependents. The Post-9/11 GI Bill was officially enacted on August 1, 2009, resulting in a $42 \%$ increase in military enrollment in higher education from 2009 and 2010 (National Conference of State Legislatures, 2014). In addition to the Post-9/11 GI Bill, other laws and VA programs have sought to increase access to post-

secondary education for veterans: Montgomery GI Bill; Survivors' and Dependents’ Educational Assistance (SDEA); Post-Vietnam Era Veterans Education Assistance Program (VEAP); Reserve Educational Assistance Program (REAP); and the Veterans Retraining Assistance Program (VRAP) (Department of Veterans Affairs, 2014). 
The number of Post-9/11 veterans is expected to rise in the coming years as servicemembers return from combat and active-duty service. There were 2.6 million Post-9/11 veterans in the United States in 2014, a figure that is anticipated to increase by $46 \%$ to 3.5 million by 2019 (Department of Veterans Affairs, 2016). Between 2000 and 2012, 900,000 veterans utilized educational benefits under the Post-9/11 GI Bill (National Conference of State Legislatures, 2014). While current data suggests that student veterans comprise only $4 \%$ of university students nationwide, this number is expected to rise as greater numbers of servicemembers return from OEF and OIF and assimilate into civilian life (National Conference of State Legislatures, 2014).

Terminology. This study utilized terminology recommended by Barry et al. (2014). Barry et al. (2014) suggested the most common terms used in studies on SSM/V (e.g., "student veterans" and "military undergraduates") are "inadequate and noninclusive" (p. 31). The researchers further argued that the umbrella term of "student veterans" fails to include those individuals who are currently serving in the Reserves or the National Guard (i.e. active duty servicemembers). Finally, the term "military undergraduates" is inadequate because it does not include graduate students and those individuals pursuing other degree programs like an associate's degree or technical certification. In the current study, for example, 23 participants self-identified as graduate or professional students (22.3\%) and three participants self-identified as distance learners (2.9\%).

Barry et al. (2014) suggested that future studies utilize the term "student servicemembers and veterans" (SSM/V) to foster inclusivity and consistency across the literature. Based on these suggestions, this study used SSM/V to describe current and former military servicemembers in higher education. This term includes veterans, active duty servicemembers, and reservists in the 
Army, Navy, Marine Corps, Air Force, and Coast Guard; commissioned officers of the Public Health Service (PHS); and commissioned officers of the National Oceanic and Atmospheric Association (NOAA) (Dortch, 2014). This definition was used in the current study to distinguish SSM/V from civilian (i.e., non-military) students.

Demographics. Post-9/11 veterans comprise a diverse group of individuals. For one, they are the youngest cohort of veterans currently served by the VA, representing a median age of 33 years old (Department of Veterans Affairs, 2016). Compared to all other veterans in 2014, Post-9/11 veterans were significantly more likely to identify as non-white, single, and uninsured. Further, they were more likely than all other veterans to have no income and to live in a household that receives food stamps (Department of Veterans Affairs, 2016). The Department of Veterans Affairs (2016) reported other notable differences between Post-9/11 veterans and their non-veteran counterparts. For example, Post-9/11 veterans were more likely than non-veterans to identify as non-white, divorced, and insured. The Post-9/11 cohort was also significantly less likely to live in poverty and more likely to have a higher personal income compared to nonveterans.

Diversity in terms of demographics among Post-9/11 veterans is represented throughout the SSM/V population. Previous literature strongly supports that SSM/V are distinctly different from their civilian (i.e., non-military) peers (Barry et al., 2014). A systematic review of the literature based on 13 empirical studies revealed several domains that separate SSM/V from civilian students (Barry et al., 2014). Of these investigations, nine studies used quantitative methodology, like the current study, and four used qualitative methodology. SSM/V reported a significantly lower Grade Point Average (GPA); riskier health behaviors; lower peer support; and differing motivations concerning alcohol use compared to civilian students (Barry et al., 
2014). Barry et al. (2014) also concluded that SSM/V participants in all of the selected qualitative studies emphasized the extent to which they differed from their non-military peers across several domains including confidence, needs, maturity, expressed difficulties, and experiences.

SSM/V comprise a nontraditional student group. Similar to other nontraditional students, SSM/V typically do not enter postsecondary education immediately following high school and are often independent of their parents or primary caretakers. Approximately $47 \%$ of all SSM/V have a family of their own including a spouse and/or children (National Conference of State Legislatures, 2014). Undergraduate SSM/V seniors, especially those exposed to combat, are significantly more likely than non-military students to identify as first generation; a distance learner; a transfer student; male; African American; older; and/or disabled (National Survey of Student Engagement, 2010). While only comprising $15.1 \%$ of active duty personnel, females account for 27\% of SSM/V nationwide (Department of Defense, 2014; National Conference of State Legislatures, 2014). According to the National Conference of State Legislatures (2014), $\mathrm{SSM} / \mathrm{V}$ comprise $4 \%$ of college students nationwide.

Despite shared values and beliefs, the military is highly diverse in terms of individual experience. Military servicemembers and veterans are likely to endorse differing experiences based on time and place of service; type of unit (e.g., fighter squadron); branch of service; and/or occupation (Hsu, 2010). They also comprise a diverse group across multicultural domains including race, ethnicity, religious affiliation, age, gender, marital status, sexual orientation, military cohort, and socioeconomic status (Department of Veterans Affairs, 2016; Kennedy et al., 2007). Similar to clinical practice with other special populations, mental health providers are 
faced with the inevitable challenge of balancing a knowledge of military culture with a sensitivity to individual experience.

Military culture. Multicultural counseling is widely accepted as a critical competency in psychological research and practice (American Psychological Association, 2002). Considering the increasing number of SSM/V on today's college campuses, multicultural counseling competence within college student counseling warrants knowledge of specific issues relevant to SSM/V, not to mention an understanding of military culture. It is important for college and university administrators, especially those in college student counseling, to be mindful of military culture and its potential influence on acculturation, adjustment, and subsequent help-seeking among SSM/V.

While both environments are steeped in tradition, cultural differences between the military and postsecondary education can be especially challenging for SSM/V. The military and higher education extol different values, perhaps as a result of their differing purposes. For example, the United States Armed Forces (USAF) was established to protect our country. Characterized by a highly structured environment, the military emphasizes values surrounding chain-of-command, sacrifice, honor, traditional notions of masculinity, and duty. On the other hand, higher education is a broad term that encompasses a range of institutions including universities, colleges, seminaries, academies, vocational colleges, and institutes of technology. In general, higher education lacks the clear boundaries and rigidity offered by a military environment. Facets of military culture include self-discipline; professional ethos; ceremony and etiquette; and cohesion (Dunivin, 1997; Hsu, 2010).

Historically speaking, the military promotes traditional notions of masculinity. Dunivin (1997) wrote, "Soldiering was viewed primarily as a masculine role because combat has 
generally been defined as men's work. Consequently, a deeply entrenched cult of masculinity (i.e. masculine norms, values, and lifestyles) pervades U.S. military culture" (p. 2). This belief system includes values traditionally associated with a machismo paradigm: conservatism; moralism; a warrior mentality; exclusion; homogeneity; separatism; and hostility (Dunivin, 1997).

Considering the aforementioned cultural differences, the transition from the military to higher education may prove to be especially difficult for some SSM/V as they renegotiate their identities from service member to student (Rumann \& Hamrick, 2010). Other adjustment issues include negative interactions with institutional personnel (e.g., university agents are perceived as unsympathetic or lacking necessary knowledge); lack of tracking resources; barriers to the identification of student veterans; transfer credits; funding and receipt of benefits; inherent cultural differences; and personal responsibilities (Griffin \& Gilbert, 2015; Olsen, Badger, \& McCuddy, 2014).

In a qualitative study on student veterans' adjustment to higher education, Livingston (2009) found that student veterans tend to camouflage their veteran status as a means of blending in and avoiding any attention their military service may bring. The researcher wrote,

The profound difference between the institutions of higher education and the military created culture shock for re-enrolling student veterans. Participants explained they had to adjust from a rigid, structured, and regimented work environment to a routine which was much more varied and flexible. (Livingston, 2009, p. 184).

Livingston (2009) found that student veterans described themselves as more mature, disciplined, and academically focused than their non-military college peers. Results from the study also suggested that self-reliance is an important coping strategy for student veterans, a value derived 
from military service. The researcher noted that while self-sufficiency contributed to the participants' individual success, this quality also “catalyzed reluctance to utilizing coping resources" (Livingston, 2009, p. 180). Unfortunately, the research surrounding coping resources among SSM/V is limited. This study sought to address this issue by examining psychological help-seeking as a coping strategy among SSM/V.

Research has demonstrated that male gender is negatively associated with psychological help-seeking (Topkaya, 2014). That said, additional research is necessary to determine whether gender is a significant predictor of psychological help-seeking among SSM/V. While machismo values appear to be deeply entrenched in military culture, a shift in ideology also seems to be taking place. Dunivin (1997) suggested this evolving paradigm includes cultural variables such as tolerance, egalitarianism, heterogeneity, and inclusion. In general, these values appear to align with belief systems within higher education. While research demonstrates that SSM/V are distinct from their non-military peers (Barry et al., 2014), it is unclear whether acculturation into higher education may involve a paradigm shift with regards to psychological help-seeking. Furthermore, a shift in ideological paradigm, group norms, and belief systems that guide military culture could impact how current and former servicemembers perceive psychological treatment, including SSM/V.

\section{Mental Health}

Existing research has indicated specific mental health needs among SSM/V (e.g., Bonar et al., 2014; Rudd, et al., 2011; Schonfeld et al., 2015). For example, Schonfeld et al. (2015) found that $28.3 \%$ of SSM/V reported adjustment difficulties upon leaving the military and entering higher education. Individuals with adjustment difficulties $(n=49)$ reported higher levels of depression and PTSD, in addition to lower levels of psychological health, than those 
who denied having adjustment difficulties (71\%). Furthermore, research has shown that student veterans report clinically elevated levels of generalized anxiety and suicidal risk (Rudd et al., 2011). Relevant literature has demonstrated that psychological concerns among SSM/V are not significantly different than those reported by non-student military populations (Bonar et al., 2014). These concerns include depression, anxiety, high-risk drinking, and post-traumatic stress disorder (PTSD).

Post-traumatic stress disorder. A considerable body of literature has shown a high rate of PTSD symptoms in SSM/V, particularly among those individuals exposed to combat. For example, Rudd et al. (2011) reported significantly elevated levels of combat exposure and PTSD among SSM/V, a prevalence rate that is consistent with other empirical investigations concerning SSM/V (e.g., DiRamio et al., 2008; Love, Levin, \& Park, 2015). Campbell and Riggs (2015) also examined the psychological, relational, and academic functioning of SSM/V ( $n=117)$. The results revealed that $25.6 \%$ of the sample reported clinically elevated PTSD symptoms based on the Impact of Event Scale-Revised (IES-R).

Combat-exposure. There is a need for psychological services among military populations, especially among those individuals who have engaged in combat-duty. Mental health problems such as PTSD, traumatic brain injury (TBI), major depression, and generalized anxiety are significantly related to combat exposure; psychological referral and treatment utilization; and attrition from military service (Hoge, Auchterlonie, \& Milliken, 2006). The prevalence of self-reported mental health problems among combat-exposed members is $19.1 \%$ for those returning from OIF in Iraq; 11.3\% of those returning from OEF in Afghanistan; and $8.5 \%$ for those returning from other combat locations (Hoge et al., 2006). 
In a systematic review of the literature, Barry and Wadsworth (2014) concluded that combat-exposed SSM/V were more likely than other SSM/V to experience PTSD, psychosomatic symptoms, suicidal thoughts, and relationship strains. These individuals are also more likely to engage in high-risk health behaviors including excessive alcohol use (Barry, Whiteman, Wadsworth, \& Hitt, 2012). Love et al. (2015) also examined the perceptions of SSM/V in terms of campus climate and combat-recovery. Among a sample of SSM/V $(n=189)$, $36.1 \%$ of participants reported high levels of stress; $15.1 \%$ reported high levels of anger; $17.3 \%$ reported current PTSD symptoms; and $27.1 \%$ screened positive for alcohol issues.

DiRamio et al. (2008) explored the experiences of student veterans, all of whom were deployed at least once between 2003 and 2007. The findings revealed several overarching challenges faced by SSM/V participants including financial barriers (e.g., balancing work and school) and lack of academic preparation (e.g., poor study habits and decreased concentration). DiRamio et al. (2008) found that in general, psychological concerns including anger management and PTSD were challenging aspects of the transition from military service to higher education for the participants.

Suicidality. Previous literature has examined suicidality among SSM/V. Rudd et al. (2011) found that $46 \%$ of student veterans $(n=525)$ reported suicidal thinking in the past. Overall, $20 \%$ of the student veterans reported suicidal thoughts with a plan; $10.4 \%$ reported considering suicide often or very often; $7.7 \%$ reported a suicide attempt; and $3.3 \%$ reported that a suicide attempt was likely or very likely. These rates are higher than the general student population in which $6 \%$ of university (i.e., non-military) students reported "seriously considering suicide" and $1.3 \%$ of university students reported a suicide attempt (American College Health Association, 2011). 
Consistent with Rudd et al. (2011), Bryan and Bryan (2015) found a high rate of suicidality among SSM/V $(n=422)$. With regards to lifetime incident rates, the results indicated that $33.4 \%$ of SSM/V reported ideation; $13.7 \%$ reported ideation with a plan; and $6.9 \%$ reported a suicide attempt. For incident rates within the last year, $14.7 \%$ of SSM/V reported ideation; $3.6 \%$ reported a plan; and $0.7 \%$ reported an attempt. Finally, within the last month, $7.6 \%$ reported ideation; $1.9 \%$ reported a plan; and $0.5 \%$ reported an attempt. These rates, while alarming, are lower than in those published in Rudd et al. (2011). This discrepancy could be explained by the samples used by the researchers. Rudd et al. (2011) focused on veterans, while Bryan and Bryan (2015) included both current and former servicemembers.

Social support. Research has demonstrated that a strong support network can foster academic success and positive adjustment among SSM/V (Love et al., 2015). According to Love et al. (2015), SSM/V who have social support report significantly lower levels of stress, anger, and interpersonal conflicts. Further, the results demonstrate that a majority of SSM/V endorsed hopefulness about the future (85.7\%) and satisfaction with university life (76\%). Further, two thirds of the SSM/V sample endorsed using adaptive coping strategies to manage stress including watching television and movies; listening to music; seeking interpersonal and romantic relationships; exercising; reading; studying; and avoiding drugs and alcohol. Consistent with previous literature, social connectedness can be a strong protective factor against the development of psychological disorders (Romero, Riggs, \& Ruggero, 2015). Relevant to this study, SSM/V may benefit from additional support from university counseling centers.

\section{Psychological Help-Seeking}

Health service psychologists and other mental health professionals are well aware of the potential benefits of psychological treatment. However, reluctance among consumers to seek 
services can be a significant challenge in the prevention and treatment of psychological disorders (Rickwood \& Thomas, 2012). Rickwood et al. (2005) defined help-seeking as, "behaviour of actively seeking help from other people" (p. 4). Psychological help-seeking, therefore, is an extension of this process that involves a person's willingness to engage in psychological or psychiatric treatment. Based on previous literature, it appears that several factors can impact psychological help-seeking: attitudes toward help-seeking (Topkaya, 2014); self-concealment (Kelly \& Achter, 1995); gender (Topkaya, 2014); mental health stigma (Held \& Owens, 2012; Topkaya, 2015); attachment style (Shaffer, Vogel, \& Wei, 2006; Vogel \& Wei, 2005); and emotional openness (Vogel, Wade, \& Hackler, 2008).

While outcome research has shown that psychotherapy is an effective intervention across a broad range of mental health concerns (Wampold, 2001), there is an underutilization of psychological services among adults aged 18 or older in the United States (Baron, Lattie, Ho, \& Mohr, 2013). Corrigan (2004) noted, "Despite the plethora of evidence-based interventions, researchers are familiar with two disconcerting trends vis-a`-vis effective treatment: (a) many people with mental illness never pursue treatment, and (b) others begin treatment but fail to fully adhere to services as prescribed" (p. 614). In 2012, 43.7 million adults (13.9\% of the total population) reported a mental, behavioral, or emotional disorder. However, only 17.9 million adults with a psychological disorder received mental health treatment in the prior year (National Institute of Mental Health Services, 2013). Data from this report suggest that many adults with psychological concerns do not, in fact, seek treatment.

An underutilization of psychological services should not be confused with a lack of interest. For example, Baron et al. (2013) found high interest but low utilization of mental health services and behavioral medicine counseling among adults in the United States. Results 
indicated that $45.7 \%$ of the sample demonstrated interest in mental health services but only $21.3 \%$ actually sought treatment. However, the findings did reveal that participants who reported mental health concerns (i.e., anxiety or depression) were significantly more likely than other patient subgroups (e.g., overweight or obese patients, smokers, and chronic pain patients) to endorse psychological help-seeking.

Previous literature has examined reasons for low rates of treatment utilization among potential consumers. According to a report conducted by the National Institute of Mental Health Services (2013), the top three reasons for underutilization were: (1) cost of care; (2) lack of knowledge concerning where to seek services; and (3) the belief that psychological issues could be resolved without formal treatment. Among a sample of university students, Topkaya (2015) found that social stigma, reluctance to share issues with a stranger, the belief that psychological issues can be resolved without formal treatment and a lack of knowledge surrounding the helping process were primary barriers to psychological help-seeking. Despite the aforementioned barriers, several factors can facilitate psychological help-seeking, including increased availability of psychological services, a belief in the benefits of psychological treatment, client-therapist trust and low-cost treatment (Topkaya, 2015).

Mental health stigma. The relationship between stigma and psychological help-seeking has been widely examined in the literature among college and military populations. An extensive body of research indicates that negative beliefs (i.e., stigma) towards receiving mental health services are a significant barrier to psychological treatment in both of these populations (e.g., Corrigan, 2004; Pattyn, Verhaeghe, Sercu, \& Bracke, 2014; Topkaya, 2014, 2015; Vogel, Wade, \& Haake, 2006). Mental health stigma can perpetuate harmful stereotypes, prejudice, and 
discrimination that can negatively influence a person's quality of life and wellness (Corrigan, 2004).

There are several mental health stereotypes that may deter individuals from engaging in psychological treatment. For example, stereotypes commonly, though inaccurately, represent consumers as incompetent, psychotic, violent, and/or at fault for their condition (Corrigan, 2004). Additionally, mental health concerns are often seen as synonymous with psychosis (Fuller, Edwards, Procter, \& Moss, 2000). Fuller et al. (2000) found that despite a need for mental health services, rural community members were reluctant to seek treatment because, "mental health problems were equated with insanity" (p. 150). The assumption that consumers are "psychotic" is not limited to rural communities. Rather, this notion remains normalized in more developed parts of the world, including the United States. Left unchallenged, such stereotypes can promote stigmatizing messages that cause people to avoid psychological treatment.

Types of stigma. The literature has focused on two types of mental health stigma: public-stigma and self-stigma (Corrigan \& Penn, 1999; Corrigan \& Watson, 2002). Publicstigma refers to the beliefs and assumptions that society deems socially acceptable toward people with mental health concerns. For example, society may perceive people with psychological issues as dangerous (Corrigan, 2004). Fuller et al. (2000) noted, "The conventional understanding of mental health problems as implying irremediable insanity leads to fear about what happens to people who become clients within the mental health care system" (p. 151). Individuals with unmet mental health concerns may fail to engage in treatment to avoid the harmful consequences of being labeled as socially undesirable or inept (Corrigan, 2004; Topkaya, 2014; Vogel et al., 2006). 
Self-stigma is when an individual internalizes society's misconceptions about mental health treatment (Corrigan, 2004). Vogel et al. (2006) defined self-stigma as the "reduction of an individual's self-esteem or self-worth caused by the individual self-labeling herself or himself as someone who is socially unacceptable" (p. 325). Self-stigma can lead to decreased self-esteem and self-efficacy and negative emotions including shame, anger, and depression on the part of the stigmatized individual (Corrigan, 1998). While public-stigma is negatively associated with psychological help-seeking, the literature has suggested that it is not a significant predictor of help-seeking (Topkaya, 2014). Extant literature suggests that self-stigma mediates the significant association between public-stigma and psychological help-seeking (e.g., Blais \& Renshaw, 2014; Held \& Owens, 2012; Vogel, Schectman, \& Wade, 2010).

College populations. Existing research has explored the constructs of stigma and psychological help-seeking in college student populations (e.g., Eisenberg, Speer, \& Hunt, 2012; Pederson \& Vogel, 2007; Topkaya, 2014). Topkaya (2014) found that male gender and selfstigma were significant predictors of help-seeking attitudes among college students at a Turkish institution. These predictors accounted for $24 \%$ of the variance within the regression model. The results corroborated with previous research suggesting that males hold more negative attitudes toward psychological help-seeking than females (Vogel, Heimerdinger-Edwards, Hammer, \& Hubbard, 2011).

Results from the Topkaya (2014) study demonstrated that while public-stigma was associated with psychological help-seeking, it was not a significant predictor of psychological help-seeking attitudes. The researcher concluded that self-stigma is more important in predicting attitudes toward psychological help-seeking than public-stigma among college students. These results are consistent with findings from Lally, O'Conghaile, Quigley, Bainbridge, and 
McDonald (2013). Lally et al. (2012) found that public-stigma was not a significant predictor of help-seeking intentions among a university student sample. Similar to Topkaya (2014), results from Lally et al. (2013) suggested that public-stigma was negatively correlated with help-seeking among students at an Irish university.

Findings from the literature have been inconsistent in terms of the prevalence of selfstigma among college students. For example, Eisenberg et al. (2012) found that $65 \%$ of college students ( $n=2,350)$ with untreated depression, anxiety, or suicidal ideation reported low-stigma and positive attitudes about psychological treatment effectiveness. Overall, these findings suggest that psychological help-seeking may be increasingly more accepted among college students. Unlike military populations, knowledge and attitudes regarding psychological treatment may no longer be a perceived barrier to psychological help-seeking among college populations. Perhaps, higher education promotes a culture of acceptance that counters mental health stigmatization.

Military populations. The construct of psychological help-seeking has been studied among military populations. Based on a sample of active-duty and veteran servicemembers, Held and Owens (2012) concluded that self-stigma negatively influenced participants' attitudes toward seeking psychological services. Similarly, Ouimette et al. (2011) found that discomfort to seek help and fear of negative social consequences were stigma-related barriers to treatment for veterans. The results from Held and Owens (2012) and Ouimette et al. (2011) were consistent with results from other investigations on psychological help-seeking among college students.

In addition to self-stigma, servicemembers may experience anticipated enacted stigma. This type of stigma is similar to public-stigma. Anticipated enacted stigma is a service 
member's belief that "they will experience hostility, discrimination, or negativity from others if their treatment seeking is made known" (Blais \& Renshaw, 2014, p. 114). A discussion about anticipated enacted stigma among military populations requires an understanding of military culture. Given the inherent danger of service, the military has an important stake in a service member's wellness, including their psychological fitness. As a matter of precaution and protection, servicemembers are not be privy to the same level of privacy as their civilian counterparts. As a result, members may not seek necessary help because they do not want to experience negative career consequences as a result of being perceived as unfit for duty (Held \& Owens, 2012).

Blais and Renshaw (2014) examined self-stigma and anticipated enacted stigma among a sample of 138 Iraq and Afghanistan servicemembers. The researchers found that self-stigma and anticipated enacted stigma among servicemembers were positively correlated. Furthermore, these two types of stigma were negatively correlated with psychological help-seeking. However, after accounting for self-stigma, anticipated enacted stigma was not a significant predictor of psychological help-seeking.

Cultural norms and values are factors that can strongly contribute to an individual's attitudes toward psychological help-seeking (Fuller et al., 2000). For example, Fuller et al. (2000) found that rural and remote communities discourage psychological help-seeking due to a deeply entrenched culture surrounding self-reliance, a value that contradicts the relational and interpersonal nature of psychological treatment. Similarly, the military promotes traditional gender norms, toughness, and self-reliance, values that may discourage members from engaging in psychological help-seeking. 
Ultimately, the more a service member endorses self-stigma, the less likely they are to seek necessary psychological help (Blais \& Renshaw, 2014). Blais and Renshaw (2014) concluded that help-seeking interventions with military populations should focus on reducing self-stigma among servicemembers. Findings from Blais and Renshaw (2014) align with results from Kim et al. (2011), in which self-stigma was negatively associated with psychological helpseeking. Similarly, Held and Owens (2011) explored the relationships among public-stigma, self-stigma, and psychological help-seeking among 126 active-duty and veteran servicemembers. Consistent with previous literature, the findings revealed that self-stigma fully mediated the association between public-stigma and psychological help-seeking.

Student servicemembers and veterans. Bonar et al. (2014) found moderate levels of mental health utilization among SSM/V, including their use of VA services. Among the sample of SSM/V $(n=81), 46.9 \%$ of the participants reported seeking mental health treatment over the last year. The findings revealed no significant differences in terms of utilization based on demographics (e.g., gender, age, and race/ethnicity) and presenting concerns (e.g., depression and high-risk drinking). Students who endorsed clinically elevated psychological concerns (i.e., depression, anxiety, and PTSD) but did not engage in treatment indicated several barriers to treatment: documentation of treatment in military records (42.9\%); embarrassment (31\%); concerns about treatment harming one's career (28.6\%); confidentiality (28.6\%); difficulty with scheduling an appointment (28.6\%); and being perceived as weak as a result of treatment (26.2\%). Stigma reduction in terms of psychological help-seeking may be especially important for SSM/V as they "have become accustomed to a military culture that emphasizes selfsufficiency and eschews weakness" (Bonar et al., 2014; p. 444). 
Recent data suggest that military students are, in fact, utilizing college counseling services, though the details are limited. Between 2013 and 2014, college counseling administrators $(n=499)$ reported that military students comprised an average of $3.59 \%$ of the student body and an average of $2.45 \%$ of the total number of clients served at the counseling centers (Reetz, Krylowicz, \& Mistler, 2014). In addition, 62 administrators indicated that specific steps were taken to assist SSM/V, the most frequent responses being to collaborate with Veterans Affairs (VA) offices and to work alongside multicultural offices to promote counseling services specifically to SSM/V.

Gender. While suicide rates are highest among males (National Institute of Mental Health, 2013), males are also far less likely than females to seek necessary psychological treatment (Komiya et al., 2000). Despite demonstrated need, research indicates that males utilize psychological services at a significantly lower rate than females (Komiya, Good, \& Sherrod, 2000; Vogel, Wester, Hammer, \& Downing-Matibag, 2014). Several reasons have been cited in the literature for reduced psychological help-seeking among males: to avoid talking about distressing circumstances (Vogel \& Wester, 2003); to avoid hurtful emotions (Komiya et al., 2000); and to mitigate social stigma (Deane \& Chamberlain, 1994).

Another reason widely cited for low utilization among males is that psychological helpseeking conflicts with traditional Western notions of masculinity (Pederson \& Vogel, 2007; Vogel et al., 2011). For men, psychological help-seeking may be synonymous with weakness, an idea that contradicts traditional gender roles. This conflict has been widely examined in the literature through the construct of gender-role conflict (GRC; O'Neil, 1981a; 1981b; 2008). GRC is defined as "the psychological state in which socialized gender roles have negative consequences for the person or others" (O'Neil, 2008, p. 362). O'Neil, Good, and Holmes 
(1995) indicated that GRC is most apparent in patriarchal societies in which adherence to traditional gender roles result in the restriction and devaluation of a person's human potential. Rigid gender roles can result in problems across four relevant domains, including cognition (i.e., how one thinks about gender roles); affect (i.e., how one feels about gender roles); unconscious experience (i.e., how gender role dynamics in life outside their conscious awareness); and behavior (i.e., how one acts as a result of gender roles) (O’Neil, Helm, Gable, David, \& Wrightsman, 1986; O’Neil et al., 1995).

Toughness (i.e., suppression of outward expression of emotion) is central to masculine norms in most Western societies (Jakupcak, Blais, Grossbard, Garcia, \& Okiishi, 2014). Traditional gender roles dictate that men are supposed to control their emotions and issues, thereby discouraging the expression of vulnerability such as fear, anxiety, or worry and encourage extreme self-reliance (Thompson \& Pleck, 1986). While toughness may be an adaptive coping mechanism in combat settings, this construct may be maladaptive in nonmilitary environments like higher education. Among a sample of Iraq and Afghanistan veterans, for example, higher levels of emotional toughness were positively associated with the likelihood for self-reported PTSD and depression (Jakupcak et al., 2014). That said, males, or females who highly ascribe to emotional toughness, may not seek necessary help due to traditional gender norms relevant to toughness and self-reliance.

The literature has also examined how males navigate tension related to gender norms and masculine ideology, especially concerning psychological help-seeking (Jakupcak et al., 2014). For some males, especially those in the military, psychological help-seeking may be perceived as a deviation from gender norms. Traditional notions of masculinity emphasize physical and psychological toughness and discourage emotional vulnerability. The literature has 
demonstrated that male military students report higher levels of authoritarianism and traditional gender perceptions than males at civilian institutions (Robinson, Kurpius \& Lucart, 2000). Since therapy involves emotional vulnerability and self-disclosure, the act of receiving mental health services could be seen as a weakness among individuals who highly ascribe to masculine ideology. Traditional masculine gender norms affirm that males should be dominant, stoic, controlled, and self-reliant. Males who highly conform to these values have a reduced likelihood of engaging in psychological help-seeking (Mahalik et al., 2003)

\section{Summary}

While the literature related to military and college counseling is vast and growing, there is a gap in knowledge concerning the psychological help-seeking experiences of SSM/V. Existing data has indicated rising numbers of SSM/V on today's college campuses (National Conference of State Legislatures, 2014), though research on psychological help-seeking among this population remains limited. Since previous studies have focused on psychological helpseeking among military and college populations, it is fitting that future studies would also examine this construct among SSM/V. Ultimately, this line of research could provide valuable information on variables that impact mental health treatment, especially within college student counseling. Considering the growing number of SSM/V on today's college campuses and research supporting specific mental health needs within this population (Griffin \& Gilbert, 2015; National Conference of State Legislatures, 2014), research concerning the experiences of SSM/V, especially surrounding psychological help-seeking, is timely and warranted.

As discussed, previous research has examined psychological help-seeking among college and military populations (e.g., Held \& Owens, 2012; Topkaya 2014). Overall, the literature has suggested that self-stigma and male gender are negative predictors of psychological help-seeking 
in both military and college populations. However, prior to the current study, gender, stigma, and psychological help-seeking have yet to be examined among SSM/V. In addition to being well-situated within the counseling psychology literature, future studies on psychological helpseeking and SSM/V would contribute to adequately preparing postsecondary institutions, especially university counseling centers, to address the needs of this student group.

\section{Research Questions}

The current study expanded on previous findings by focusing on psychological helpseeking among the SSM/V population. This study sought to contribute to the increasing discourse and empirical interest on SSM/V, and to further situate this topic within the counseling psychology literature. Based on a thorough review of the selected literature, this study specifically examined whether gender, self-stigma, and public-stigma significantly predicted psychological help-seeking among SSM/V. Thus, this study sought to address the following research questions:

1. Does self-stigma as measured by the Self-Stigma of Seeking Help Scale (Vogel et al., 2006) predict attitudes toward psychological help-seeking as measured by the Attitudes Toward Seeking Professional Psychological Help Scale - Short Form (ATSPPH-SF; Fischer \& Farina, 1995) in SSM/V?

2. Does public-stigma as measured by the Stigma Scale for Receiving Psychological Help (Komiya et al., 2000) predict attitudes toward psychological help-seeking as measured by the ATSPPH-SF (Fischer \& Farina, 1995) in SSM/V?

3. Is there a significant difference in attitudes toward psychological help-seeking as measured by the ATSPPH-SF (Fischer \& Farina, 1995) between males and females? 
4. Does the combination of predictor variables (gender, self-stigma, and publicstigma) significantly predict attitudes toward psychological help-seeking as measured by the ATSPPH-SF (Fischer \& Farina, 1995) in SSM/V?

\section{Hypotheses}

In light of previous literature and based on the aforementioned research questions, the following hypotheses were established for the current study:

H1. Self-stigma as measured by the Self-Stigma of Seeking Help Scale (Vogel et al., 2006) will significantly predict attitudes toward psychological help-seeking as measured by the ATSPPH-SF (Fischer \& Farina, 1995) in SSM/V.

H2. Public-stigma as measured by the Stigma Scale for Receiving Psychological Help (Komiya et al., 2000) will not significantly predict attitudes toward psychological helpseeking as measured by the ATSPPH-SF (Fischer \& Farina, 1995) in SSM/V.

H3. There will be a significant difference between males and females in terms of attitudes toward psychological help-seeking as measured by the ATSPPH-SF (Fischer \& Farina, 1995).

H4. Gender as measured by the Demographics Data Form will be a significant predictor of attitudes toward psychological help-seeking as measured by the ATSPPH-SF (Fischer \& Farina, 1995) in SSM/V. 


\section{Chapter 2: Research Design and Methodology}

The primary aim of this study was to examine the extent to which self-stigma, publicstigma, and gender predicted attitudes toward psychological help-seeing among student servicemembers and veterans (SSM/V; Barry et al., 2014). Based on previous findings (e.g., Held \& Owens, 2012; Topkaya, 2014), it was hypothesized that self-stigma and gender would significantly predict psychological help-seeking attitudes in SSM/V. It was also hypothesized that public-stigma would not significantly predict psychological help-seeking attitudes in SSM/V. Finally, it was hypothesized there would be a significant difference in attitudes toward psychological help-seeking between males and females. This chapter will provide an overview of the methodology used to address the stated research questions and hypotheses.

Methodological details will be provided in terms of the research design, participants, instrumentation, recruitment, procedures, and data analysis.

\section{Research Design}

The present study employed a cross-sectional research design in which the relationships between and among the variables were analyzed across a representative subset of the SSM/V population at a particular point in time. In contrast to an experimental design, the researcher did not form a subject group a priori and no variables were manipulated in the context of the current study. Rather, self-report data from the preexisting treatment or control group (i.e., SSM/V) was examined to formulate a predictive model of psychological help-seeking. This study used a cross-sectional design in which the individual scores of the participants in the subject group were compared to one another.

In the current study, the selected research design enabled the researcher to define and describe the nature of the relationships between and among the predictor variables (self-stigma, 
public-stigma, and gender) and criterion variable (psychological help-seeking attitudes). This design also allowed the researcher to effectively describe, explain, and predict the characteristics of psychological help-seeking in SSM/V, an important outcome of scientific exploration (Greenberg, 1986). Data for the current study was collected through Qualtrics, an online platform for conducting survey research. The use of a survey research provided several advantages: ease of data collection, cost-effectiveness, and usefulness in describing relationships among complex variables in the selected population (Heppner, Kivlighan, \& Wampold, 1999). All participants in the present study were administered the same self-report questionnaire via Qualtrics.

\section{Data Analysis}

The data was analyzed using the IBM Statistical Package for the Social Sciences (SPSS) Statistics (2015) program. Relative to the current study, the SPSS software program has the capacity to conduct descriptive statistics, bivariate statistics, parametric tests, and predictive analysis. Demographic information was collected for each participant using the Demographics Data Form. Further, data analysis was conducted using a hierarchical multiple regression test and independent samples t-test. Assumptions for each statistical test were evaluated prior to analysis. $\mathrm{H}_{3}$ was tested using an independent samples t-test. This statistical test determined whether there were significant differences in the mean scores on the Attitudes Toward Seeking Professional Psychological Help Scale - Short Form (ATSPPH-SF; Fischer \& Farina, 1995) between males and females. Hierarchical multiple regression was used to address RQ1, RQ2, and RQ4, in addition to $\mathrm{H}_{1}, \mathrm{H}_{2}$, and $\mathrm{H}_{4}$. This analysis provided a systematic way of determining whether the three predictor variables significantly predicted the criterion variable.

\section{Participants}


Participants were recruited through email. The target population for this study included active-duty and former military servicemembers who were currently enrolled in a degree-seeking program. An a priori power analysis was conducted using G*Power 3 (Faul, Erdfelder, Lang, \& Buchner, 2007) to determine an appropriate sample size. This analysis was computed using a small effect size of 0.15 (Cohen, 1988), three predictor variables (self-stigma, public-stigma, and gender), and a statistical power of 0.80 . The selected effect size and statistical power were consistent with previous studies (e.g., Topkaya, 2014). In addition, Heppner et al. (1999) indicated a power of 0.80 is the accepted standard in counseling research. Results from the a priori power analysis revealed that a total sample size of $77 \mathrm{SSM} / \mathrm{V}$ participants was adequate for this study. Therefore, the researcher sought to collect data from at least 77 participants, but no more than 150 participants. This limit was based on previous literature in which Baruch (1999) indicated an average response rate of 55.6\% $(S D=19.7)$ in academic studies. More recently, Baruch and Holtom (2008) indicated an average response rate of 52.7\% $(S D=20.4)$. For this reason, a limit of 150 participants was established in order to account for an estimated response rate of $51.3 \%$.

Criteria. A few criteria were required for participation in the current study. All participants had to be adults who were 18 years of age or older. Additionally, participants had to be currently enrolled in a degree-seeking program and had to be current or former military servicemembers. This included veterans, active duty servicemembers, and reservists in the Army, Navy, Marine Corps, Air Force, or Coast Guard; commissioned officers of the Public Health Service (PHS); and commissioned officers of the National Oceanic and Atmospheric Association (NOAA) (Barry et al., 2014; Dortch, 2014). Finally, while the Post 9/11 GI Bill and 
other educational programs offer benefits to dependents (i.e. spouses and children of servicemembers), dependents were excluded from participation included in this study.

Sampling procedure. While random sampling would have been an ideal technique in this study, this method is impractical and thus, seldom used in counseling research (Heppner et al., 1999). Therefore, convenience sampling was used in the current study. Convenience sampling allowed the researcher to recruit a subject group based on proximity and ease-of-access (Urdan, 2010). This study was conducted at a large, public, land-grant university in the Appalachian region of the Mid-Atlantic during the spring 2017 semester. The institution had a total enrollment of 28,487 students, including 14,702 males (52\%) and 13,785 females (48\%), in Fall 2016. Based on data collected from the College Board's Annual Survey of Colleges (2016), the racial and ethnic profile of the student body is as followed: 79\% White/Caucasian; 6\% Nonresident International Student; 5\% Black/African American; 4\% Two or More Races; 4\% Hispanic/Latino/a; and 2\% Asian American/Asian. In fall 2017, there were 396 SSM/V currently enrolled at the university, including 60 females (15\%) and 336 males (85\%) (J. McCarthy, personal communication, September 8, 2017). Considering these demographics, this institution was an ideal setting for the current study given its proximity and potential access to $\mathrm{SSM} / \mathrm{V}$.

\section{Variables}

Three predictors were assessed including self-stigma, public-stigma, and gender. The first two predictors, self-stigma and public-stigma, are continuous variables that were assessed according to the Self-Stigma of Seeking Help Scale (Vogel et al., 2006) and the Stigma Scale for Receiving Psychological Help (Komiya et al., 2000). These variables were operationalized according to previous studies (e.g., Corrigan 1998, 2004; Corrigan \& Watson, 2002; Topkaya, 
2014; Vogel et al., 2006). In the current study, self-stigma was defined as the adoption of negative attitudes toward oneself based on seeking psychological treatment (Corrigan \& Watson, 2004). Self-stigma is an extension of the broader construct of public-stigma. Public-stigma was defined as the adoption of negative attitudes toward seeking psychological help based on society's negative attitudes toward individuals with mental health concerns. Ultimately, the literature indicates that negative perceptions held by society as a whole can perpetuate stereotypes and assumptions towards people who engage in psychological help-seeking (Corrigan 1998, 2004). Vogel et al. (2006) noted that public-stigma can result in seeing a person who seeks psychological help as socially inept or undesirable. The third predictor analyzed in this study was gender. Gender was assessed according to self-report data in the demographics portion of the questionnaire. For the purposes of the current study, gender was understood as the state of being male or female based on the participant's own assessment of their gender identity.

The three predictor variables were measured in relation to one criterion, or outcome, variable. The criterion variable was attitudes toward psychological help-seeking as measured by the Attitudes Toward Seeking Professional Psychological Help Scale - Short Form (ATSPPHSF; Fischer \& Farina, 1995). Psychological help-seeking was defined as the act of pursuing mental health support from other people (Rickwood et al., 2005). While help-seeking can include both informal and formal sources of help, this study focused on formal help such as psychotherapy or psychiatric treatment. A participant's attitudes toward psychological helpseeking was defined as the degree to which they perceived psychological or psychiatric treatment as positive or negative.

\section{Measures}


The present study examined the relationship between and among three predictor variables (self-stigma, public-stigma, and gender) relative to unique variability in one criterion variable (attitudes toward psychological help-seeking). Several instruments were used to measure these variables: a Demographics Data Form; the Self-Stigma of Seeking Help Scale (Vogel et al., 2006); Stigma Scale for Receiving Psychological Help (Komiya et al., 2000); and the Attitudes Toward Seeking Professional Psychological Help Scale - Short Form (Fischer \& Farina, 1995).

Demographics Data Form. A Demographics Data Form was administered to all participants in the subject group at the onset of the questionnaire (APPENDIX C). Participants were asked to disclose several pieces of information: gender; race/ethnicity; age; year in school; institution; type of degree program (e.g., bachelor's degree, associate's degree, graduate degree, etc.); branch of military service; veteran or active-duty status; and number of years of service. In addition, the demographic form assessed whether the participant had participated in combat and if so, inquired further about their combat experience (i.e., number of deployments to a conflict zone and which conflict they served in).

Self-Stigma of Seeking Help Scale (SSOSH; Vogel et al., 2006). The SSOSH was used to measure self-stigma among the sample. The SSOSH measures the degree to which a person perceives seeking psychological services as harmful to one's self-satisfaction, self-confidence, and overall worth as a human being. This scale has been used to measure self-stigma in college students (Topkaya, 2014) and military servicemembers (Held \& Owens, 2012). This Likert-type scale is comprised of 10 items with response options of (1) strongly disagree, (2) disagree, (3) agree and disagree equally, (4) agree, and (5) strongly agree with a total possible score of 50. Higher scores on the SSOSH are indicative of higher levels of self-stigma towards psychological 
help-seeking. Results from the current study demonstrated an average SSOSH score of 26.17 $(S D=7.30)$.

Example statements on the SSOSH include, "I would feel inadequate if I went to a therapist for psychological help," "My self-esteem would increase if I talked to a therapist," and "If I went to a therapist, I would be less satisfied with myself." Vogel et al. (2006) reported a strong internal consistency reliability of $0.91(n=583)$ and test-retest reliability of $0.72(n=226)$ after two months, indicating the instrument possesses good test-retest reliability over a twomonth time interval. The researchers showed evidence for negative associations between SSOSH scores and the Attitudes Toward Seeking Professional Psychological Help Scale ( $r=-$ $.63, p<.01)$. In other words, individuals with higher levels of self-stigma are more likely to have negative attitudes toward seeking psychological services. In the present study, the SSOSH demonstrated slightly less, though still adequate, internal reliability $(\alpha=0.86)$.

Stigma Scale for Receiving Psychological Help (SSRPH; Komiya et al., 2000). The SSRPH was used to measure public-stigma among the sample. The SSRPH measures the degree to which a person perceives psychological treatment as stigmatizing. Komiya et al. (2000) developed the scale using a sample of undergraduate students $(n=311)$. This scale has been used to measure public-stigma in college students (Topkaya, 2014) and military servicemembers (Held \& Owens, 2012). The Likert-type scale is comprised of five items rated on a 4-point scale including (0) strongly disagree, (1) disagree, (2) agree, and (3) strongly agree with a total possible score of 20. Higher scores on the SSRPH are indicative of higher levels of perceived stigma towards psychological help-seeking (i.e., public stigma). Results from the current study demonstrated an average SSRPH score of $12.35(S D=2.99)$. 
Example statements on the SSRPH include, "People tend to like less those who are receiving professional psychological help" and "It is advisable for a person to hide from people that he/she has seen a psychologist." The SSRPH has a coefficient alpha of 0.72 , indicating an adequate internal consistency (Komiya et al., 2000). While a higher coefficient alpha is desirable in terms of internal consistency, a coefficient alpha of 0.72 meets the standard minimum of 0.70 in social science research (Kline, 1999). The SSRPH (Komiya et al., 2000), a measure of public-stigma, demonstrated acceptable internal reliability $(\alpha=0.78)$. In the present study, the SSRPH demonstrated similar internal reliability $(\alpha=0.78)$.

Komiya et al. (2000) showed evidence for the instrument's construct validity through the use of factor analysis. Results from the factor analysis suggested the existence of one factor. Once the first factor was removed, Komiya et al. (2000) noted a large eigenvalue discontinuity. This factor accounted for an estimated 100\% of the variance in SSRPH among the tested population. Furthermore, Komiya et al. (2000) reported a strong negative correlation between the SSRPH and the Attitudes Toward Seeking Professional Psychological Help Scale ( $r=-.40, p$ $<.01)$. Individuals who endorsed higher levels of public-stigma also endorsed more negative attitudes toward psychological help-seeking. Consistent with the literature, Komiya et al. (2000) indicated that females reported significantly lower levels of public-stigma compared with males.

\section{Attitudes Toward Seeking Professional Psychological Help Scale - Short Form}

(ATSPPH-SF; Fischer \& Farina, 1995). The ATSPPH-SF was used to measure attitudes toward psychological help-seeking among the sample. The Attitudes Toward Seeking Professional Psychological Help Scale (Fischer \& Turner, 1970) and ATSPPH-SF (Fischer \& Farina, 1995) are regarded as the most widely used standardized psychometric instruments for assessing mental health treatment attitudes (Elhai, Schweinle, \& Anderson, 2006). The literature 
has demonstrated strong psychometric support for both of these measures (Elhai et al., 2006; Fischer \& Farina, 1995; Vogel, Wester, Wei, \& Boysen, 1995). The ATSPPH-SF (Fischer \& Farina, 1995) is the contemporary adaptation of the ATSPPH (Fischer \& Turner, 1970). The ATSSPH and ATSPPH-SF are intended to clarify a person's attitudes toward seeking or resisting professional mental health services during times of psychological crisis or distress.

The ATSPPH-SF was used in this study to measure participants' attitudes toward seeking mental health services. The ATSPPH-SF has been used in previous research with college students (Topkaya, 2012) and military servicemembers (Held \& Owens, 2012). This 10-item scale is a modification of the original 29-item scale (Fischer \& Turner, 1970). Statements on the ATSPPH-SF are rated using a 4-point Likert scale with corresponding scores including (3) agree, (2) partly agree, (1) partly disagree, and (0) disagree with a total possible score of 30 . Higher scores on the ATSPPH-SF are associated with less treatment stigma and greater intentions to seek mental health services in the future. Results from the current study demonstrated an average SSOSH score of $16.50(S D=6.03)$.

Example statements on the ATSPPH-SF include, "I would want to get psychological help if I were worried or upset for a long period of time" and "The idea of talking about problems with a psychologist strikes me as a poor way to get rid of emotional conflicts." Fischer and Farina (1995) reported a strong internal consistency reliability coefficient of 0.84 . The researchers found evidence of the instrument's validity by point-biserial correlations between respondents who had sought help and those who had not ( 0.39 overall, 0.24 for women, and 0.49 for men). In the present study, the ATSPPH-S demonstrated slightly better internal reliability ( $\alpha$ $=0.85)$.

\section{Procedures}


As discussed, a quantitative research design was implemented in this study using a selfreport questionnaire. A Qualtrics survey was made available online through a hyperlink embedded in the recruitment email. Emails were sent to the Veterans Advocate and president of the student veterans organization requesting they distribute the recruitment letters to SSM/V at the university. Recruitment emails were then forwarded to members of the student organization and to students registered with the Office of Veterans Affairs through the Veterans Advocate and president of the student veterans organization. The president of the student veterans organization also posted a blurb about the study on the organization's Facebook page.

The recruitment email included a link to the Qualtrics survey, in addition to information about participation requirements, informed consent, risks and benefits, estimated time for completion, incentives, and contact information (APPENDIX A). The recruitment email also provided details about an incentive drawing. Participants were informed of the opportunity to enter to win one of six $\$ 25.00$ electronic Amazon gift cards. They were informed that they had the opportunity to provide their email address at the end of the survey to enter the drawing. Subjects were informed that providing an email address was voluntary, that their email address would not be associated with their individual responses, and that all email addresses would be stored in a separate password-protected document. Upon closing the survey, six email addresses were selected using Random Picker, an online service for conducting random drawings. These individuals were each individually emailed a $\$ 25$ electronic gift certificate.

Once participants read the recruitment letter and selected the hyperlink to the Qualtrics survey, they were brought to a webpage displaying the informed consent (APPENDIX B). The informed consent included basic information about the study including contact information for the researchers, Institutional Review Board (IRB) protocol number, and study title. The body of 
the informed consent provided participants with details about the study including an introduction, purpose statement, deidentification procedures, description of the procedures, discomforts, benefits, financial considerations, and voluntary participation statement. Subjects were informed of the participation requirements and asked whether they agreed to the informed consent. If the participant did not meet the minimum requirements or declined participation, they were directed to the end of the survey where they were thanked for their participation and provided with a list of mental health resources (APPENDIX G). All members of the subject group had to agree to the informed consent before proceeding with the questionnaire.

After participants agreed to the informed consent, the Demographics Data Form was displayed (APPENDIX C). This form collected demographic information from the participants, as outlined in the Measures section. After the demographics form, the remaining instruments were displayed including the SSSHS (Vogel et al., 2006; APPENDIX D), SSRPH (Komiya et al., 2000; APPENDIX E), and the ATSPPH-SF (Fischer \& Farina, 1995; APPENDIX F). To reduce the negative effects of priming, the order of the four instruments was randomly displayed to participants. After completing the questionnaire, participants were given the opportunity to provide their email address to enter the incentive drawing. Upon finishing the survey in its entirety, participants were directed to a webpage thanking them for their participation. Furthermore, a list of mental health resources targeted towards military servicemembers were provided to all participants. 


\section{Chapter 3: Results}

\section{Demographics}

Data for the present study was collected during the spring 2017 semester. The participant sample $(n=103)$ was comprised of SSM/V currently enrolled in a degree-seeking program including 26 females $(25.2 \%)$ and 77 males (74.8\%). The gender distribution of the sample was consistent with national data that indicates that females represent $27 \%$ of all SSM/V in the United States (Department of Defense, 2014; National Conference of State Legislatures, 2014). Responses were classified as complete if they offered valid responses to the Demographics Data Form; Self-Stigma of Seeking Help Scale (SSOSH; Vogel et al., 2006); Stigma Scale for Receiving Psychological Help (SSRPH; Komiya et al., 2000); and Attitudes Toward Seeking Professional Psychological Help Scale - Short Form (ATSPPH-SF; Fischer \& Farina, 1995). Participants who did not complete all the measures in their entirety $(n=15)$ were excluded from the study.

In terms of racial and ethnic identity, participants described themselves as White/Caucasian (88.3\%; $n=91)$, Multiracial $(5.8 \% ; n=6)$, Black/African American $(1.9 \% ; n=$ 2), Hispanic/Latino/a (1.9\%; $n=2)$, Asian American/Asian $(1 \% ; n=1)$, and Did Not Specify $(1 \% ; n=1)$. The average age of the sample was 28.96 years old $(S D=8.23)$ with a range of 19 to 54 years old. Participants completed an average of 6.94 years of military service $(S D=6.05)$ with a range of 1 to 30 years of service. The sample consisted of 10 freshmen $(9.7 \%), 17$ sophomores (16.5\%), 19 juniors (18.4\%), 30 seniors (29.1\%), 23 graduate/professional students (22.3\%), three distance learners (2.9\%), and one did not specify (1\%). Participants were asked to provide information about their military branch and combat status. Most of the participants were Army servicemembers $(49.5 \% ; n=51)$ and most identified themselves as veterans $(59.2 \% ; n=$ 
61). Table 1 provides a visual representation of the military and combat status for the participant sample.

Table 1

Demographics by Combat and Military Status

\begin{tabular}{lcc}
\hline Variable & $\begin{array}{c}\text { Number of } \\
\text { Participants }\end{array}$ & $\%$ \\
\hline Military Status & 1 & 1.0 \\
Active-duty & 41 & 39.8 \\
National Guard/Reserve & 61 & 59.2 \\
Veteran & & \\
Military Branch & 17 & 16.5 \\
Air Force & 51 & 49.5 \\
Army & 2 & 1.9 \\
Coast Guard & 16 & 15.5 \\
Marine Corps & 13 & 12.6 \\
Navy & 2 & 1.9 \\
Commissioned Officer of the & 2 & 1.9 \\
More than one branch & & \\
Deployment to Combat/Armed & 49 & 47.6 \\
Yes & 54 & 52.4 \\
No & & \\
Deployment Location & 11 & 22.4 \\
Iraq & 16 & 32.7 \\
Afghanistan & 15 & 30.6 \\
Multiple Locations & 6 & 12.2 \\
Other & 1 & 2.0 \\
Did Not Specify & &
\end{tabular}

Note. $n=103$

\section{Coding and Scoring}

Gender was dummy coded $(0=$ male, $1=$ female $)$. Scores were calculated for the SSOSH (Vogel et al., 2006), SSRPH (Komiya et al., 2000), and ATSPPH-S (Fischer \& Farina, 1995). The SSOSH measures perceptions of self-stigma. Responses to items 2, 4, 5, 7, and 9 were reversed scored. Individual responses were then totaled to produce a composite score where higher scores represent greater perceptions of self-stigma. The average self-stigma score 
was $26.17(S D=7.30)$. The SSRPH measures perceptions of public-stigma where higher scores reflect greater perceptions of public-stigma. Total public-stigma scores were calculated by totaling the responses to each individual item on the test. The mean public-stigma score was $12.35(S D=2.99)$. Finally, attitudes toward psychological help-seeking were measured using the ATSPPH-SF with higher scores indicating more positive attitudes toward professional help. First, reverse scoring was conducted for items $2,4,8,9$, and 10 . Next, a total score was computed for each participant by summing their responses to each individual item. The average attitudes toward psychological help-seeking score among participants was $16.50(S D=6.03)$.

\section{Internal Consistency of Measures}

Internal consistency, or internal reliability, is thought to measure general agreement among a set of items that propose to measure the same construct. Cronbach's alpha was calculated for each scale to measure the internal consistency of the instrument. The internal consistency for each scale ranged from "acceptable" to "good" based on Cronbach's alpha (George \& Mallery, 2003). The SSOSH (Vogel et al., 2006), a measure of self-stigma, had good internal reliability $(\alpha=0.86)$. The SSRPH (Komiya et al., 2000), a measure of public-stigma, demonstrated acceptable internal reliability $(\alpha=0.78)$. Finally, the ATSPPH-S (Fischer \& Farina, 1995), a measure of attitudes toward psychological help-seeking, had a good internal reliability $(\alpha=0.85)$. Since the alpha score for each instrument demonstrated at least acceptable internal reliability, all scales were analyzed in their entirety as detailed in Chapter 2.

\section{Statistical Assumptions}

Prior to conducting the multiple regression analysis, the completed responses were evaluated across six assumptions: (1) independence of observations; (2) linearity; (3) homoscedasticity; (4) multicollinearity; (5) no significant outliers, high leverage points, or highly 
influential points; and (6) normal distribution of residual errors. The assumption of independence of observations was met according to a Durbin-Watson test statistic of 1.521. This assumption was met because the Durbin-Watson test statistic was between 1.5 and 2. (Field, 2013). The assumption of linearity was tested by plotting the studentized residuals and the unstandardized predicted variables on a scatterplot (Figure 1). A visual inspection of the scatterplot indicated that the residuals formed a horizontal band which suggested a linear relationship between the dependent variable and predictor variables. Further, since the spread of residuals did not significantly increase or decrease across the scatterplot (Figure 1), the assumption of homoscedasticity was also met. The assumption of multicollinearity was met by default because this study had only one dependent variable (attitudes toward psychological helpseeking). There were no outliers in the sample based on an examination of the studentized deleted residuals in the dataset, all of which were within \pm 3 standard deviations (Field, 2013). No leverage points were observed as the leverage values were less than 0.2. Further, no influential points were found in the sample because the Cook's Distance values were less than 1.0 (Cook \& Weisberg, 1982). Finally, a scatterplot (Figure 2) revealed an approximately normal distribution of the standardized residuals. To confirm these findings, a P-P plot (Figure 3) was generated revealing that the assumption of normality was met because the residuals were approximately normally distributed along the diagonal line.

Four assumptions were checked before conducting the independent samples t-test: (1) independence of observations; (2) no significant outliers, high leverage points, or highly influential points; (3) approximately normal distribution of predictor variables; and (4) homogeneity of variances. The independence of observations test was met because there were different participants in each gender group. A boxplot (Figure 4) was generated and upon visual 
inspection, it was determined there were no outliers in the dataset. Further, psychological helpseeking scores were normally distributed for the male group $(p=.329)$ and female group $(p=$ .329) based on the Shapiro-Wilk's test. Finally, a Levene's test revealed that the assumption of homogeneity of variances was met $(p=.994)$.

Figure 1

Scatterplot of Studentized Residuals and Unstandardized Predicted Values

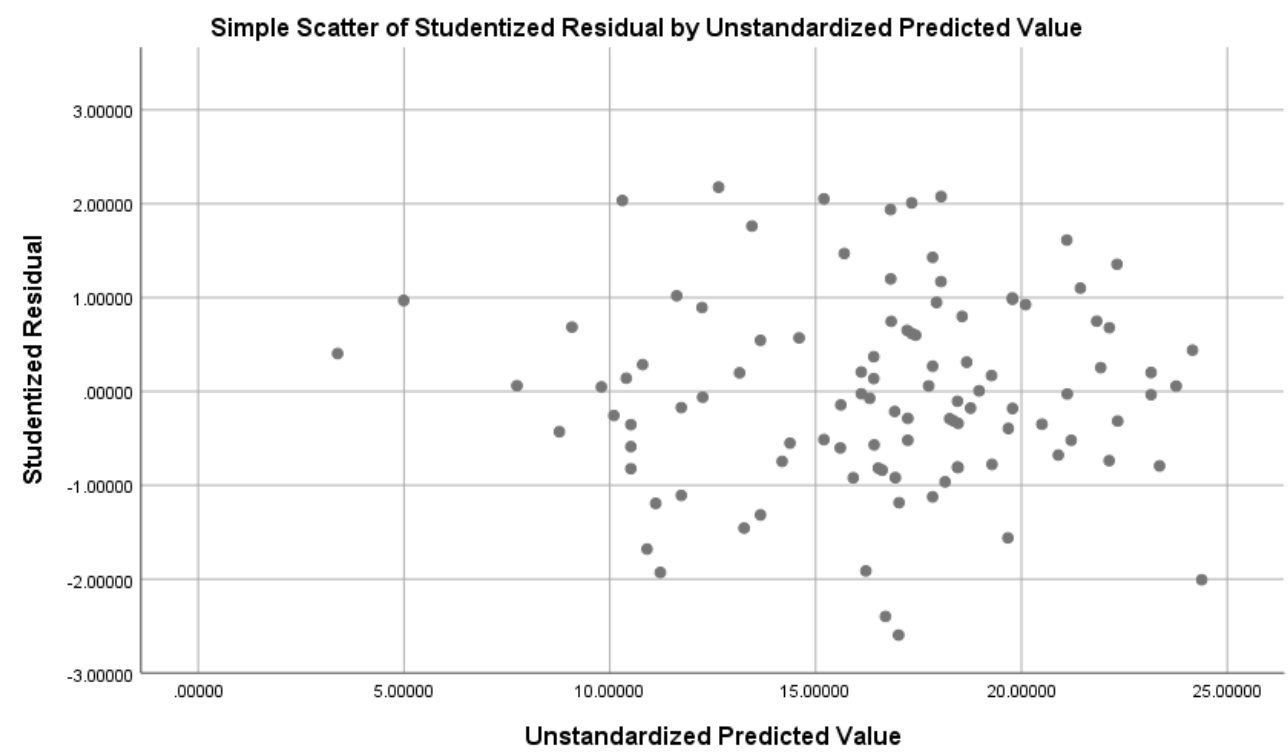

Figure 2

Standardized Residual Histogram of Psychological Help-Seeking Score

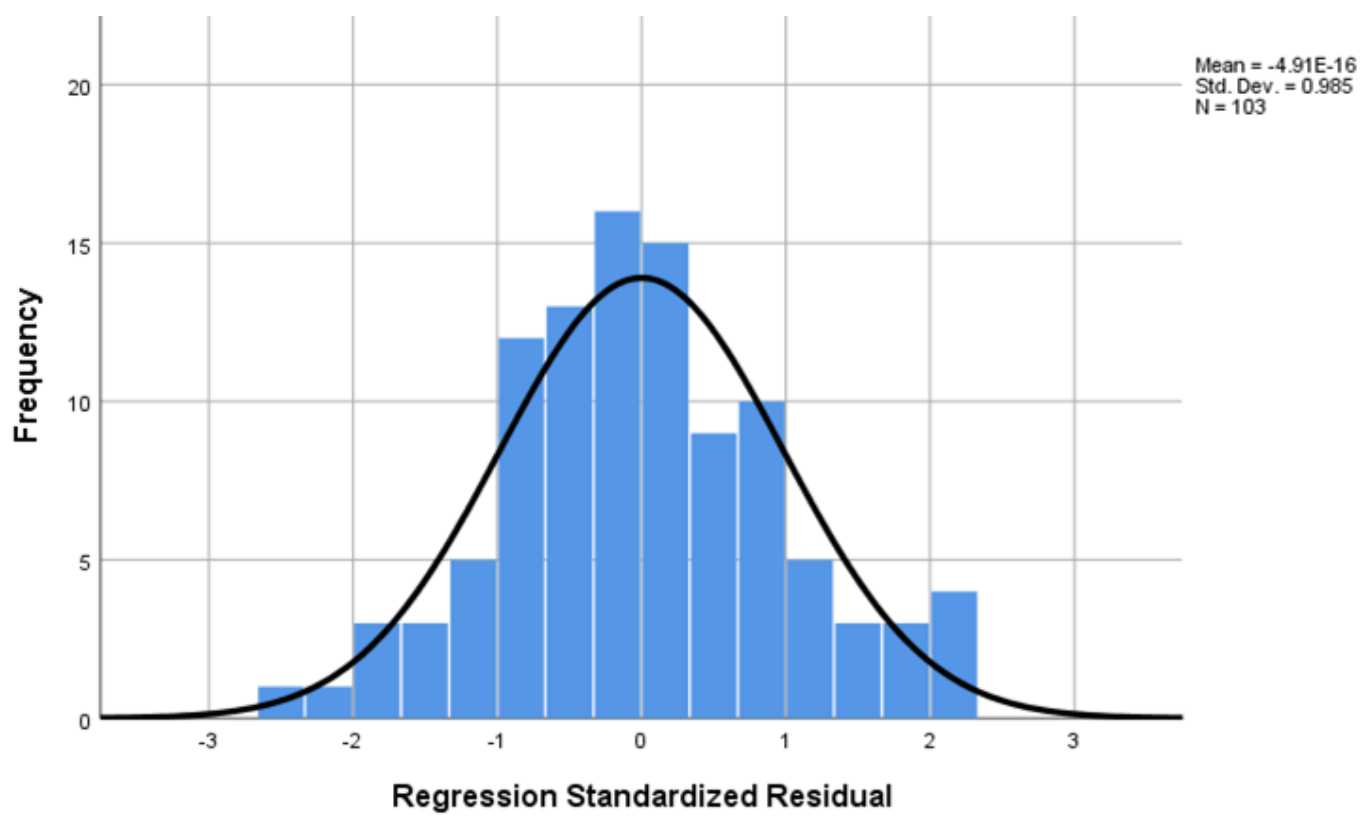


Figure 3

P-P Plot of Regression Standardized Residual

Normal P-P Plot of Regression Standardized Residual

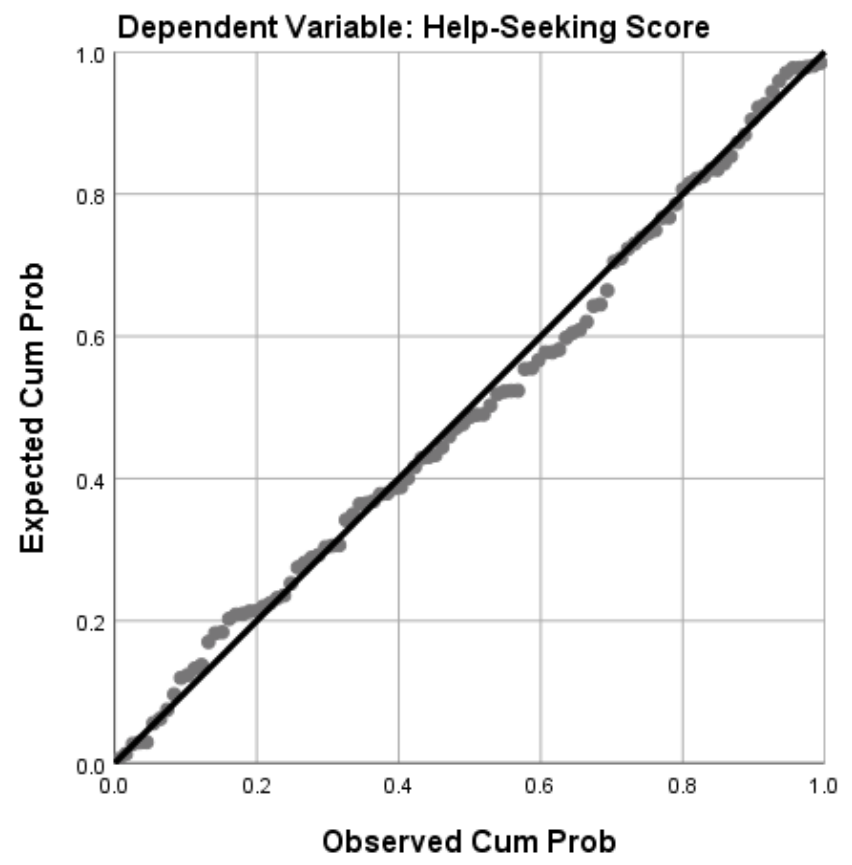

Figure 4

Boxplot of Psychological Help-Seeking Scores by Gender

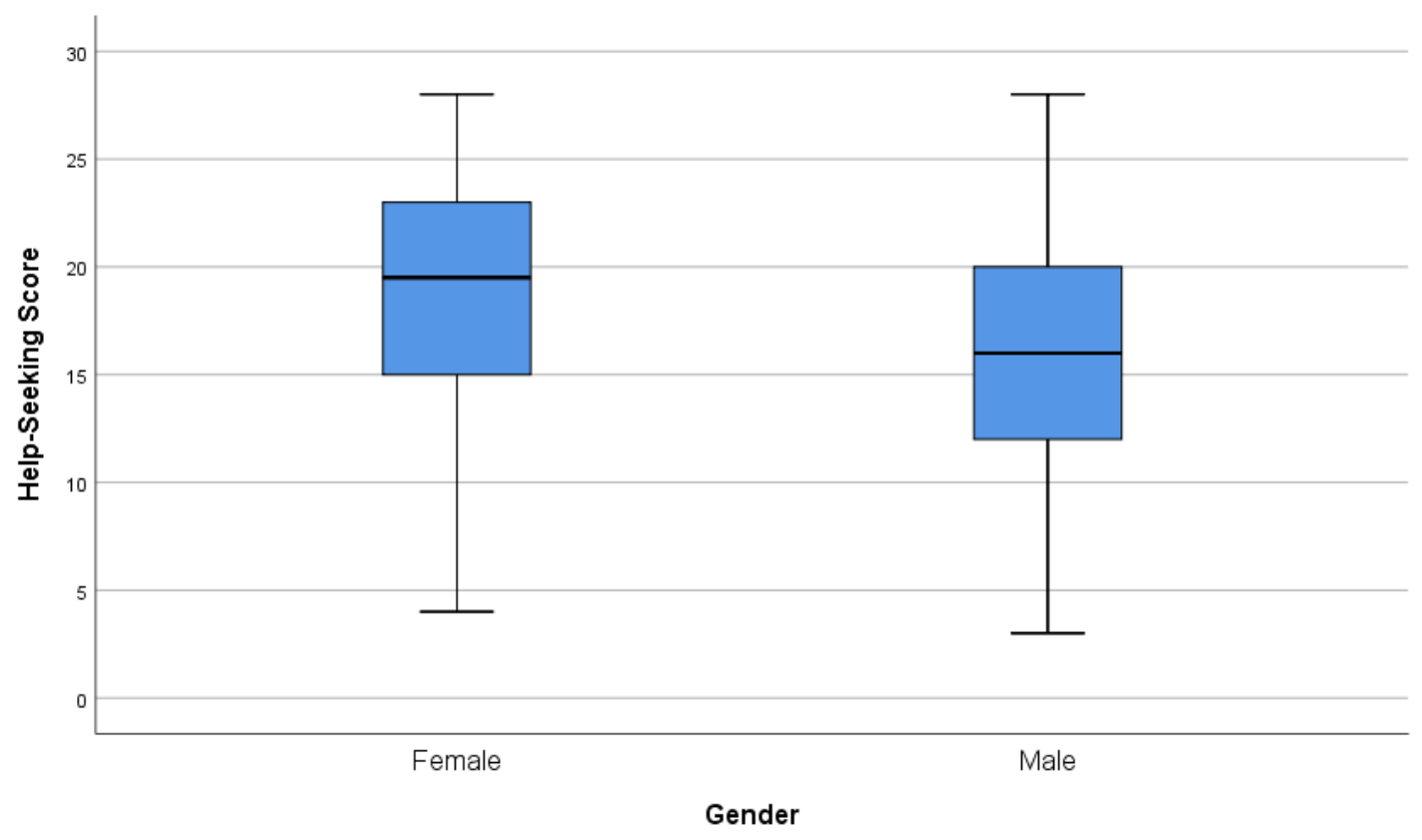




\section{Multiple Regression Analysis}

A multiple regression analysis was conducted to evaluate the individual and collective predictive strength of the three predictors in relation to the outcome variable (Heppner et al., 1999). This analysis was also used to test $\mathrm{H}_{1}, \mathrm{H}_{2}$, and $\mathrm{H}_{4}$. Hierarchical methodology was used to build the overall regression model. Since the literature surrounding SSM/V is limited, the data was not entered based on an underlying theory. Instead, the predictors were entered based on their respective correlations with attitudes toward psychological help-seeking. Hierarchical methodology provided the advantage of a systematic process for entering the predictors into the model, in addition to identifying those factors that accounted for the most unique variance in the criterion variable (Heppner et al., 1999).

Pearson correlation analysis was used to determine the effect size of each predictor relative to the criterion (Table 2). Guidelines provided by Cohen (1988) were used to interpret the effect sizes. Results from the Pearson correlation analysis indicated statistically significant relationships between each predictor and the criterion. There was a large negative effect for selfstigma and attitudes toward psychological help-seeking, $r(101)=-.653, p<.01$. Greater perceptions of self-stigma were associated with more negative attitudes toward psychological help-seeking. There was a moderate negative effect for public-stigma and attitudes toward psychological help-seeking, $r(101)=-.440, p<.01$. Greater perceptions of public-stigma were associated with more negative attitudes toward psychological help-seeking. Finally, there was a small negative effect for gender and attitudes toward psychological help-seeking, $r(101)=-.198$, $p=.023$. Results from the independent samples t-test revealed that females endorsed significantly more positive attitudes toward psychological-help-seeking than males. 
Based on hierarchical methodology, each predictor was successively entered in the model based on the strength of its relationship with the outcome variable. Self-stigma, the predictor with the strongest correlation, was entered in the first step of the model $(r=-0.653)$. Publicstigma, the predictor with the second strongest correlation $(r=-0.440)$, was entered in the second step of the model. Finally, gender, the predictor with the weakest correlation $(r=-0.198)$, was entered in the third step of the analysis.

Table 2

Correlations Among Possible Predictor Variables of Psychological Help-Seeking

\begin{tabular}{lcccc}
\hline & 1 & 2 & 3 & 4 \\
\hline 1. Help-Seeking & -- & & & \\
2. Self-Stigma & $* *-.653$ & -- & & \\
3. Public- & $* *_{-} .440$ & $* * .538$ & -- & - \\
Stigma & $* .198$ & -.078 & .023 & - \\
4. Gender & $*$ &
\end{tabular}

Note. One-tailed tests of significance: $* * p<.01 ; * p<.05$.

Three predictive models were assessed to evaluate the amount of unique variance explained by each predictor variable. Table 3 displays the results from each regression model generated during analysis. Supporting $\mathrm{H}_{1}$, the first three-step regression model indicated that self-stigma significantly predicted attitudes toward psychological help-seeking, $R^{2}=.426\left(\Delta R^{2}=\right.$ $.420), F(1,101)=74.998, p<.01$. Self-stigma accounted for $42 \%$ of the variance in attitudes toward psychological help-seeking where participants with greater self-stigma endorsed more negative views of psychological help-seeking and were less likely to seek psychological help ( $\beta$ $=-.653, t=-8.660, p<.01)$. Supporting $\mathrm{H}_{2}$, the addition of public-stigma $(\beta=-.125, t=-1.403$, $p=.164)$ in the second step did not account for a significant change in variance in attitudes toward psychological help-seeking, $R^{2}=.437\left(\Delta R^{2}=.011\right), F(2,100)=1.969, p=.164$. Public- 
stigma accounted for an additional $1.1 \%$ of explained variance in help-seeking. However, selfstigma remained a significant predictor of help-seeking $(\beta=-.483, t=-6.581, p<.01)$ in the second step of the regression model, with participants high in self-stigma again being less likely to seek psychological help. The overall model was also significant, $F(2,100)=38.843, p<.01$. Finally, supporting $\mathrm{H}_{4}$, the addition of gender accounted for a significant change in variance in attitudes toward psychological help-seeking, $R^{2}=.496\left(\Delta R^{2}=.059\right), F(3,99)=11.523, p<.01$. Gender $(\beta=-.3 .367, t=-3.395, p<.01)$ accounted for an additional $5.9 \%$ of explained variance, with men being less likely to seek help than women. In the third step, self-stigma ( $\beta=-.509, t=$ $-7.243, p<.01)$ also predicted help-seeking, with participants higher in self-stigma being less likely to seek help. Public-stigma ( $\beta=-.207, t=-1.207, p=.230)$ did not predict help-seeking in the third step. The full model of self-stigma, public-stigma, and gender was also significant, $F(3$, $99)=11.523, p<.01$. Together, the three predictors accounted for $49.6 \%$ of explained variance in attitudes toward psychological help-seeking. The overall predictive model of self-stigma, public-stigma, and gender in relation to attitudes toward psychological help-seeking $(y)$ produced the following equation:

$$
y=34.885-0.509 \text { (Self-Stigma) }-0.207 \text { (Public-Stigma) }-5.335(\text { Gender })
$$

\section{Independent Samples T-Test}

An independent samples t-test was conducted to test $\mathrm{H}_{3}$. This statistical test allowed the researcher to determine whether there were significant differences between males and females based on attitudes toward psychological help-seeking as measured by the ATSPPH-SF (Fischer \& Farina, 1995). Results from the independent samples t-test were evaluated to determine whether attitudes toward psychological help-seeking significantly differed between males ( $n=$ 77) and females $(n=26)$ based on self-identified gender on the Demographics Data Form. 
Supporting $\mathrm{H}_{3}$, there was a statistically significant difference in mean psychological help-seeking score between males $(M=15.81, S D=5.93)$ and females $(M=18.54, S D=5.99)$ with males scoring higher than females, $2.73 \pm 1.35, t(101)=2.028, p=.045$. In other words, males endorsed significantly more negative attitudes toward psychological help-seeking than females and thus, are less likely to seek psychological help.

Table 3

Regression Analysis of Self-Stigma, Public-Stigma, and Gender as Predictors of Help-Seeking

\begin{tabular}{|c|c|c|c|c|c|c|c|c|c|}
\hline \multirow[t]{2}{*}{ Variables } & \multicolumn{3}{|c|}{$\begin{array}{c}\frac{\text { Model 1 }}{\text { Self-Stigma on Help- }} \begin{array}{c}\text { Seeking } \\
\mathrm{R}^{2}=.426, \Delta \mathrm{R}^{2}=.426 \\
(S E=4.59)\end{array} \\
\end{array}$} & \multicolumn{3}{|c|}{$\begin{array}{l}\text { Model 2 } \\
\text { Self-Stigma and Public- } \\
\text { Stigma on Self-Stigma } \\
\mathrm{R}^{2}=.437, \Delta \mathrm{R}^{2}=.011 \\
\quad(S E=4.57)\end{array}$} & \multicolumn{3}{|c|}{$\begin{array}{c}\text { Model 3 } \\
\text { Self-Stigma, Public- } \\
\text { Stigma, and Gender on } \\
\text { Help-Seeking } \\
\\
\mathrm{R}^{2}=\begin{array}{l}.496, \Delta \mathrm{R}^{2}=.059 \\
(S E=4.35)\end{array}\end{array}$} \\
\hline & $B$ & $S E B$ & $\beta$ & $B$ & $S E B$ & $\beta$ & $B$ & $S E B$ & $\beta$ \\
\hline Self-Stigma & -.539 & .062 & $*_{-.653}$ & -.483 & .073 & $*_{-.} .586$ & -.509 & .070 & $*_{-.617}$ \\
\hline $\begin{array}{l}\text { Public- } \\
\text { Stigma }\end{array}$ & -- & -- & -- & -.252 & .180 & -.125 & -.207 & .171 & -.102 \\
\hline Gender & -- & -- & -- & -- & -- & -- & -3.367 & .992 & $*_{-.244}$ \\
\hline
\end{tabular}

Note. ${ }^{*} p<.01$

Table 4

Independent Samples T-Test of Psychological Help-Seeking Score by Gender

\begin{tabular}{lcccccc}
\hline & $n$ & $M$ & $S D$ & $t$ & $d f$ & Sig. (2 tailed) \\
\hline Females & 26 & 18.54 & 5.99 & & & \\
Males & 77 & 15.81 & 5.93 & & & \\
$\begin{array}{l}\text { Equal Variances } \\
\text { Assumed }\end{array}$ & & & & 2.028 & 101 & $.045^{*}$ \\
\hline Necen
\end{tabular}

Note. ${ }^{*} p<.01$ 


\section{Additional Analyses}

Results from the original analysis demonstrated that while public-stigma was significantly correlated with psychological help-seeking as evident in Table 2, it was no longer significant with the addition of self-stigma in Model 2 as demonstrated in Table 3. As a result, a test of mediation was conducted to assess whether self-stigma mediates the relationship between public-stigma and attitudes toward psychological help-seeking. In addition to gender $(0=$ male, $1=$ female), this mediation test was conducted using other relevant demographics (APPENDIX C) as control variables including age, race $(0=$ White, $1=$ non-White $)$, military service status $(0$ $=$ veteran, $1=$ active-duty or reservist $)$, and combat status $(0=$ never in combat, $1=$ in combat $)$ as supported by previous literature (Hoge et al. 2004; Kulesza, Pedersen, Corrigan, \& Marshall, 2015). Each variable was dummy coded accordingly. The correlations for each predictor variable are shown in Table 5.

Table 5

Correlations of Additional Possible Predictor Variables of Psychological Help-Seeking

\begin{tabular}{|c|c|c|c|c|c|c|c|c|}
\hline & 1 & 2 & 3 & 4 & 5 & 6 & 7 & 8 \\
\hline $\begin{array}{l}\text { 1. Help- } \\
\text { Seeking }\end{array}$ & -- & & & & & & & \\
\hline 2. Self-Stigma & $*_{*} *_{-} .653$ & -- & & & & & & \\
\hline $\begin{array}{l}\text { 3. Public- } \\
\text { Stigma }\end{array}$ & $*_{-} *_{-} .440$ & $* * .538$ & -- & & & & & \\
\hline 4. Gender & $* .198$ & .078 & -.023 & -- & & & & \\
\hline 5. Age & $* .239$ & $*_{-} .198$ & -.051 & $*_{-} .235$ & -- & & & \\
\hline 6. Race & .066 & -.109 & -.063 & -.002 & .094 & -- & & \\
\hline $\begin{array}{l}\text { 7. Military } \\
\text { Status }\end{array}$ & $*_{-} .230$ & .181 & -.044 & $* .200$ & $\begin{array}{l}* *_{-} \\
.606\end{array}$ & .068 & -- & \\
\hline 8. Combat & .051 & -.130 & .019 & $* *_{-} .330$ & $* * .496$ & -.164 & $\begin{array}{l}* *_{-} \\
.593\end{array}$ & -- \\
\hline
\end{tabular}

Note. Two-tailed tests of significance: $* * p<.01 ; * p<.05$. 
Consistent with the original analysis, self-stigma, public-stigma, and gender were significantly correlated with the outcome variable of attitudes toward psychological helpseeking. In addition, age and military status were also significantly correlated with psychological help-seeking. Together, the demographic variables accounted for $14.8 \%$ of the variance in attitudes toward psychological help-seeking, $R^{2}=.148\left(\Delta R^{2}=.105\right), F(5,97)=$ 3.383, $p<.01$. Results revealed a small positive effect for age and attitudes toward psychological help-seeking where increased age was correlated with more positive attitudes toward psychological help-seeking, $r(101)=.239, p=.015$. Further, there was a small negative effect for military status and attitudes toward psychological help-seeking such that active duty servicemembers and reservists endorsed more negative attitudes toward psychological helpseeking than veterans, $r(101)=-.230, p=.02$.

Results from the mediation analysis are displayed in Table 6. Using the Baron and Kenny (1986) method for testing mediation, public-stigma $(\beta=-.434, \mathrm{p}<.01)$ appeared to negatively predict attitudes toward psychological help-seeking. Next, public-stigma $(\beta=.539, \mathrm{p}$ $<.01)$ was found to positively predict self-stigma. In the third step, self-stigma $(\beta=-.636, \mathrm{p}<$ .01) negatively predicted attitudes toward psychological help-seeking. Finally, when publicstigma and self-stigma were entered in the fourth model, the relation of self-stigma $(\beta=-.562, \mathrm{p}$ $<.01)$ to psychological help-seeking remained significant and the relation of public-stigma ( $\beta=$ $.131, \mathrm{p}=.122)$ to psychological help-seeking dropped to nonsignificance. This finding suggests that self-stigma fully mediated the relationship between public-stigma and psychological helpseeking (Figure 5). In other words, the more public-stigma reported by an individual, the more self-stigma they report and in turn, the less positive views they endorse towards psychological help-seeking. 
Table 6

Self-Stigma as a Mediator between Predictor of Public-Stigma and Outcome of Help-Seeking

\begin{tabular}{|c|c|c|c|c|c|c|c|c|c|c|c|c|}
\hline \multirow[t]{3}{*}{ Variables } & \multicolumn{3}{|c|}{$\begin{array}{l}\frac{\text { Model } 1}{\text { Public-Stigma on Help- }} \\
\text { Seeking }\end{array}$} & \multicolumn{3}{|c|}{$\begin{array}{c}\text { Public-Stigma on Self- } \\
\text { Stigma }\end{array}$} & \multicolumn{3}{|c|}{$\begin{array}{l}\frac{\text { Model } 3}{\text { Self-Stigma on Help- }} \\
\begin{array}{c}\text { Seeking } \\
\text { Sekin }\end{array}\end{array}$} & \multicolumn{3}{|c|}{$\begin{array}{c}\underline{\text { Model } 4} \\
\text { Public-Stigma and Self- } \\
\text { Stigma on Help-Seeking }\end{array}$} \\
\hline & \multicolumn{3}{|c|}{$\mathrm{R}^{2}=.334(S E=5.07)$} & \multicolumn{3}{|c|}{$\mathrm{R}^{2}=.343(S E=6.11)$} & \multicolumn{3}{|c|}{$\mathrm{R}^{2}=.530(S E=4.26)$} & \multicolumn{3}{|c|}{$\mathrm{R}^{2}=.542(S E=4.23)$} \\
\hline & $B$ & $S E B$ & $B$ & $B$ & $S E B$ & $\beta$ & $B$ & $S E B$ & $\beta$ & $B$ & $S E B$ & $\beta$ \\
\hline $\begin{array}{l}\text { Public- } \\
\text { Stigma }\end{array}$ & -.875 & .169 & $*_{-.434}$ & 1.32 & .204 & $* .539$ & -- & -- & -- & -.264 & .169 & -.131 \\
\hline $\begin{array}{l}\text { Self- } \\
\text { Stigma }\end{array}$ & -- & -- & -- & -- & -- & -- & -.525 & .059 & $*_{-.636}$ & -.464 & .071 & $* .562$ \\
\hline
\end{tabular}

Note. ${ }^{*} p<.01$

Figure 5

Mediation Model with Public-Stigma, Self-Stigma, and Help-Seeking

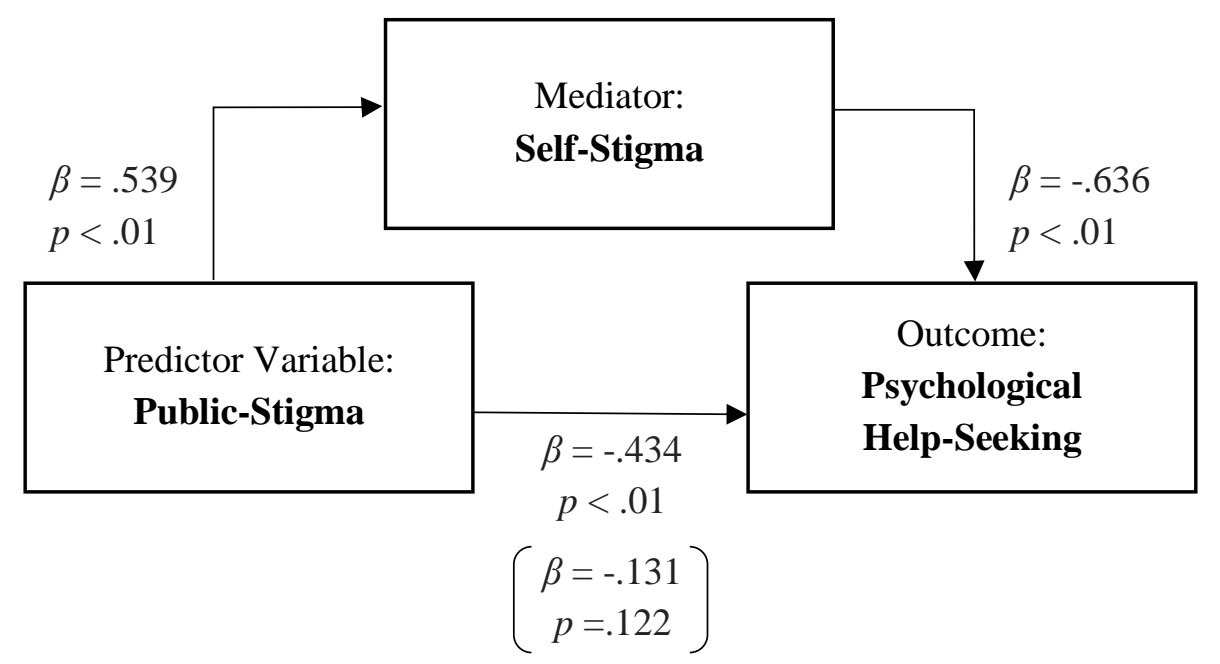

Figure 5. Model testing mediation of the relationship between public-stigma and psychological help-seeking by self-stigma. Values in parentheses represent the standardized relation of publicstigma to psychological help-seeking after controlling for self-stigma. 


\section{Chapter 4: Discussion}

The general aim of this study was to examine predictors of psychological help-seeking among student servicemembers and veterans (SSM/V). SSM/V likely experience unique challenges as they adjust from military service to student life and thus, may need specialized support from college administrators (Livingston, 2009; Niv \& Bennett, 2017). Additionally, SSM/V endorse clinically elevated mental health issues including anxiety, depression, substance use, and PTSD symptoms (e.g., Bonar et al., 2014; Rudd, et al., 2011; Schonfeld et al., 2015). While these concerns do not significantly differ from those issues reported by non-student military populations (Bonar et al., 2014), SSM/V tend to underutilize university resources (Livingston, 2009) and thus, may be less likely to seek psychological services than non-military students. Despite the growing number of SSM/V on today's college campuses (Griffin \& Gilbert, 2015; National Conference of State Legislatures, 2014), there is a gap in knowledge concerning the specific psychological needs of this population. The present study sought to address this issue by examining predictors of psychological help-seeking among SSM/V including mental health stigma and gender.

Mental health stigma is a significant barrier to psychological treatment (Barney, Griffiths, Jorm, \& Christensen, 2006). Two types of stigma, self-stigma and public-stigma, have been previously examined in the literature including among college students (e.g., Eisenberg et al., 2012; Pederson \& Vogel, 2007; Topkaya, 2014) and military populations (e.g., Blais and Renshaw, 2014; Held \& Owens, 2012; Ouimette et al., 2011). The current study sought to investigate whether self-stigma and public-stigma significantly predicted attitudes toward psychological help-seeking in a sample of SSM/V. There is strong evidence to support that selfstigma significantly predicts attitudes toward psychological help-seeking among college students 
(Topkaya, 2014) and military service members (Held \& Owens, 2012). Similarly, it was hypothesized in the current study that self-stigma would significantly predict attitudes toward psychological help-seeking in SSM/V. There is also evidence to suggest that while publicstigma is negatively associated with psychological help-seeking, it is not a significant predictor of psychological help-seeking (e.g., Held \& Owens, 2012; Lally et al., 2012; Topkaya, 2014). Therefore, it was also hypothesized that public-stigma would not significantly predict attitudes toward psychological help-seeking.

In addition to stigma, the current study investigated the effect of gender on attitudes toward psychological help-seeking. Extant literature has demonstrated that males endorse significantly more negative perceptions of psychological help-seeking than females and thus, are less likely to seek necessary psychological care (Komiya et al., 2000; Vogel et al., 2011; Vogel et al., 2014). These perceptions can be attributed to traditional belief systems surrounding masculinity (Thompson \& Pleck, 1986). It is possible that men, particularly those who highly ascribe to masculine ideals, attribute psychological treatment with emotional vulnerability and thus, weakness. In an effort to reduce social stigma, men may avoid seeking psychological help (Pederson \& Vogel, 2007; Vogel et al., 2011). Given these considerations, it was hypothesized in the current study that gender would significantly predict attitudes toward psychological helpseeking such that males would endorse significantly more negative attitudes toward psychological help-seeking than females.

Findings from the current study supported the stated hypotheses. The results revealed that self-stigma significantly predicted attitudes toward psychological help-seeking among $\mathrm{SSM} / \mathrm{V}$ where greater levels of self-stigma were associated with more negative views of psychological help-seeking. Gender also significantly predicted attitudes toward psychological 
helps-seeking. As hypothesized, males endorsed significantly more negative views toward psychological help-seeking than females. Initial results also indicated a negative association between public-stigma and attitudes toward psychological help-seeking. Similar to self-stigma, greater levels of public-stigma were correlated with more negative views of psychological helpseeking. Results from the regression model revealed a significant effect of self-stigma and gender on attitudes toward psychological help-seeking. However, the effect of public-stigma on attitudes toward psychological help-seeking disappeared upon accounting for self-stigma in the predictive model.

Additional analyses revealed that self-stigma fully mediated the significant relationship between public-stigma and psychological help-seeking. This finding corroborates with previous literature in which self-stigma has been found to mediate the significant relationship between public-stigma and psychological help-seeking among college students and military servicemembers (e.g. e.g., Blais \& Renshaw, 2014; Held \& Owens, 2012; Vogel et al., 2010). This relationship suggests that societal stereotypes about psychological help-seeking (publicstigma) are internalized by individuals (self-stigma) and thus, lead to more negative attitudes toward psychological help-seeking in SSM/V. Ultimately, these findings shed light on the process in which external events adopt internal psychological significance. Results from the current study are consistent with previous literature (Blais \& Renshaw, 2014; Lally et al., 2012; Topkaya, 2014) and thus, support the notion that mental health stigma is a barrier to psychological help-seeking for SSM/V.

There are several reasons why SSM/V, particularly men, may develop stigma and negative attitudes toward psychological help-seeking including societal expectations, military culture and identity, and vocational consequences. Attribution Theory (AT; Weiner, 1985) and 
Modified Labeling Theory (MLT; Link, Cullen, Struening, Shrout, \& Dohrenwend, 1989), provide a frame of reference for understanding how public-stigma and self-stigma impact the attitudes of SSM/V toward psychological help-seeking. AT (Weiner et al., 1985) focuses on the process in which people assign, or attribute, internal and external causes for every day events, circumstances, or behavior. Based on this theoretical perspective, it is natural for people to assign internal or external reasoning for why people struggle with mental health issues. However, this natural curiosity can lead to the perpetuation of misconceptions, stereotypes, and assumptions about individuals who struggle with mental health problems. For example, the literature indicates that individuals with mental health issues are often perceived as dangerous, unpredictable, psychotic, and responsible for their own issues (Angermeyer \& Dietrich, 2006; Brockington, Hall, Levings, \& Murphy, 1993; Fuller et al., 2000). Further, Brockington et al. (1993) indicated that people with mental health issues are habitually characterized as unable to care for themselves and frequently described as childlike or innocent. As these attributes become widely accepted, they form the basis of public stigma and result in stigmatized beliefs toward the targeted group (Corrigan, 2004; Corrigan \& Watson, 2002).

MLT (Link et al., 1989) provides a theoretical framework for understanding how these stigmatized attributes impact people's perceptions or behavior. According to this theory, people are socialized early in life to become aware of stigmatizing beliefs. Related to the current study, Angermeyer and Dietrich (2006) found that the general public holds negative views towards mental illness. As people are exposed to these beliefs, they develop a set of expectations about how someone with mental health issues will be treated by society. They may assume, for example, that the person will experience discrimination or rejection. As a result of these messages and subsequent expectations, people may grow to become resistant of psychological 
help-seeking to avoid being mislabeled as mentally unfit (Corrigan, 2004; Corrigan \& Watson, 2002). These misconceptions and stereotypes can be internalized by the individual as self-stigma such that the person may negatively judge themselves should they seek necessary psychological treatment (Vogel et al., 2006).

Results from the current study appeared to align with this theoretical conceptualization as the effect of public-stigma on attitudes toward psychological help-seeking was fully mediated by self-stigma. Based on AT (Weiner et al., 1985) and MLT (Link et al., 1989), it is possible that SSM/V adopt beliefs early in life about the personal attributes of those individuals with mental health illness. These attributes are largely negative (Angermeyer \& Dietrich, 2006; Brockington et al., 1993; Fuller et al., 2000) and may result in negative social consequences such as discrimination, judgement, or rejection. Regardless of whether these labels and consequences come to fruition, people are highly motivated to avoid the negative social implications of being labeled as psychotic or crazy (Fuller et al, 2000). Consequently, it is possible that these beliefs and assumptions lay the groundwork for the development of negative attitudes toward psychological help-seeking among SSM/V.

In addition to societal expectations, it is possible that military culture contributes to the adoption of mental health stigma among SSM/V. Therefore, the current study should be considered in light of research surrounding the lived experiences of SSM/V. Unlike traditional college students, SSM/V must navigate the challenge of balancing their identity as a student with their identity as service member. Military culture is often an important part of a student servicemember's identity (Livingston, 2009). It is possible that as members become indoctrinated into military service, they adopt a system of beliefs that are inconsistent with psychological treatment. For example, counseling services may necessitate a degree of 
emotional vulnerability that contradicts the professional ethos of self-reliance imbedded within military culture (Dunivin, 1997; Held \& Owens, 2012).

Livingston (2009) found that student veterans reported difficulties during the reenrollment process from military service to university life. For one, student veterans were less likely to seek formalized academic help due to the "lingering influence of do-it-yourself military pride" (Livingston, 2009, p. 178). The researcher wrote, "Student veterans were taught to be self-sufficient in the military, a factor which equipped them to succeed as individuals but catalyzed reluctance to utilizing coping resources" (Livingston, 2009, pp. 178-179). Similarly, the enduring influence of military culture, particularly around self-suffiency, may influence the development and expression of mental health stigma among SSM/V. Bonar et al. (2014) suggested that military culture, including an emphasis on eschewing weakness, may influence mental health utilization among SSM/V. While self-reliance is a highly adaptive quality in the military, this characteristic may result in more negative perceptions of psychological helpseeking and thus, hinder an individual from establishing necessary psychological treatment.

SSM/V may also avoid psychological help-seeking due to fear of vocational consequences (Ouimette et al., 2011) as fitness for duty is an important component of military service. Military servicemembers and veterans may seek to avoid the possibility of being perceived as unfit and thus, unable to fulfill their duties (Held \& Owens, 2012). Bonar et al. (2014) found that SSM/V who had not previously engaged in psychological services endorsed several reasons for not engaging in treatment including fear of vocational repercussions, embarrassment, and difficulty with scheduling an appointment. Bonar et al. (2014) indicated that the fear of vocational repercussions was the most cited reason for avoiding psychological treatment among SSM/V. Other reasons included wanting to avoid documentation of treatment 
in military records, concerns about harming one's career, and confidentiality issues. In addition to societal expectations and military culture, it is possible that a fear of career consequences may contribute to mental health stigma and, as supported by the current study, lead to more negative attitudes toward psychological help-seeking among SSM/V.

Finally, this study highlights the significant effect of gender on attitudes toward psychological help-seeking. Self-reliance may be both a product of military service and socially constructed ideas around masculinity. Based on AT (Weiner et al., 1985) and MLT (Link et al., 1989), it is possible that men are exposed early in life to societal expectations and beliefs about masculine attributes (Jakupcak et al., 2014). For example, young men may be socialized to believe that men should not cry and that emotional vulnerability is synonymous with weakness. These beliefs conflict with psychological treatment and ultimately, pose a gender role conflict for men (GRC; O’Neil, 2008). To negotiate this conflict and avoid negative social consequences, men may adopt a tough presentation that is consistent with masculine norms in most Western societies (Jakupcak et al., 2014). Mahalik et al. (2003) found that men who highly conform to traditional masculine attributes have a reduced likelihood of engaging in psychological helpseeking. Similarly, the current study demonstrated that males endorsed significantly more negative views of psychological help-seeking than females. Additionally, it is possible that military culture may contribute to rewarding those who exhibit traditionally masculine attributes. Robinson et al. (2000) found that male military students reported higher levels of authoritarianism and traditional gender perceptions than college men at civilian institutions. Males who highly conform to these values have a reduced likelihood of engaging in psychological help-seeking (Mahalik et al., 2003). Therefore, a mixture of both societal 
expectations and military culture may factor into the development of negative attitudes toward psychological help-seeking among SSM/V, particularly among males.

\section{Strengths}

The primary strength of this study is that it calls attention to the psychological needs of SSM/V. These results were not surprising due to existing evidence supporting that male gender and self-stigma are negatively associated with psychological help-seeking (Bonar et al., 2014; Held \& Owens, 2012; Topkaya, 2014). That said, these studies have been limited to the experiences of military and college student populations. The current study expanded on these findings by examining psychological help-seeking among military students, a historically underrepresented and underserved group. Despite the rising numbers of SSM/V on today's college campuses, Niv and Bennett (2017) found that directors at 113 university counseling centers indicated limited knowledge of service utilization among veterans. Further, Griffin and Gilbert (2015) found that institutional staff and administrators had limited methods for identifying and tracking SSM/V. The current study sought to address this gap in knowledge by examining the specific psychological needs of SSM/V. Prior to this study, it could be reasonably assumed that SSM/V face many of the same barriers to psychological treatment as military members and college students. However, this study grounds these claims in empiricism and establishes a foundation for future research on the psychological needs of SSM/V. The hope is that this study will serve as a springboard for additional research on SSM/V, especially literature that is situated in the psychological field.

\section{Limitations}

These findings and subsequent clinical implications should be considered with respect to the study's limitations. The use of a survey research design provided several advantages 
including ease of data collection, cost-effectiveness, and usefulness in describing relationships among the variables (Heppner et al., 1999). However, this research design is not without disadvantages. For one, survey research design does not establish causality (Heppner et al., 1999). For example, it is incorrect to assume that greater levels of self-stigma cause more negative attitudes toward psychological help-seeking, or vice versa. A cross-sectional research design, like the one employed in this study, provides limited information about the relationships between and among variables. Furthermore, the results from this study were based on self-report data and therefore, are inherently limited. The self-report nature of this study is limited by the possibility of engaging in the survey more than once and the potential for social desirability bias. It is important to consider the possibility that the SSM/V who elected to participate in the study may have responded to questions in way that was intended to present themselves in a favorable light.

While convenience sampling was advantageous in terms of proximity and ease of data collection, this type of sampling threatens the external validity of the findings. This study is limited in terms of its generalizability because the participants were recruited from one institution and represented a subset of the population. The participant sample was also limited in terms of diversity of military status as $49.5 \%$ identified as Army servicemembers $(n=51)$ and $99 \%$ identified as either Veteran or National Guard/Reserve $(n=102)$. Therefore, it cannot be assumed that this study's findings capture the experiences of all SSM/V at the university, nor do they represent the experiences of SSM/V in general. Thus, while the assumption of normality was met, it is important to remember that the data gathered in this study could vary from the general population of SSM/V. 
While the variables chosen for this study were based on existing literature, it is possible that other variables better predict attitudes toward psychological help-seeking among SSM/V. In this study, self-stigma and gender significantly predicted attitudes toward psychological helpseeking. It is possible that other variables, or a combination of variables, better predict the outcome variable. Therefore, self-stigma and gender are not exhaustive in terms of their predictive value. Finally, the validity and reliability of the instruments used in this study were strongly supported by the literature. However, it is possible that other instruments could better represent and measure the predictive nature of the variables.

Many of these limitations are not specific to this study but rather, a criticism of survey research in the social sciences. Like other quantitative designs in psychology, this study sought to examine complex variables through survey data, a method that typically favors simplicity and ease over complexity and depth. That said, it is important to remember that the data gathered in this study provides a snapshot of the perceptions and attitudes of a subset of the SSM/V population at a specific point in time. Considering the breadth and depth of the human experience, these findings should always be interpreted with caution.

\section{Clinical Implications}

As discussed, the results from this study suggest that self-stigma is a barrier to psychological help-seeking for SSM/V, especially among men. These findings have important implications for colleges and universities, especially those institutions that regularly cater to the needs of SSM/V. SSM/V bring diversity of experience to higher education, in addition to valuable leadership training, problem solving skills, focus, and an orientation toward servicelearning (Ang \& Kelley, 2016). Given the nature of military service, the current study sought to highlight the specialized psychological needs of this unique student group. Several practical 
recommendations are provided in this section based on these findings. These recommendations may prove to be particularly useful for institutions who hope to improve services for SSM/V.

First, it is recommended that a needs assessment be conducted to evaluate the current campus climate for SSM/V at the college or university. Considering that each campus is different, the needs assessment should lay the groundwork for programming and outreach that is tailored to SSM/V enrolled at the institution. Among other areas, the needs assessment could gather information about specific and culturally-relevant facilitators and barriers to help-seeking among SSM/V. Outside of informing short-term programming, data collected in the needs assessment might provide valuable information that could improve the pre-enrollment process for SSM/V including recruitment, admissions, and orientation (Hayden, 2014). Additionally, it could improve existing student services by tailoring programs and outreach to SSM/V and ultimately, contributing to improved retention and student engagement efforts.

Data that is gathered from the needs assessment could be used to develop a strategic plan for orienting SSM/V to civilian life and academia, in addition to increasing their awareness of student services (Hayden, 2014). Based on the results of this study, it is imperative that the plan focus on perpetuating targeted messages for SSM/V that reduce stigma and promote psychological help-seeking services. These efforts should be developed in collaboration with the institution's Veterans Affairs office. In the current study, the Veterans Advocate at the institution was an important gatekeeper in terms of identifying, recruiting, and gathering data from the university's SSM/V. Veterans Advocates can be valuable conduits through which messages that destigmatize psychological help-seeking can be effectively communicated to $\mathrm{SSM} / \mathrm{V}$. Ultimately, this collaboration can forge an important bridge between student services and SSM/V by communicating a unified message that promotes healthy coping strategies, 
reduces mental health stigma, increases awareness of services, and hopefully, facilitates access to services for SSM/V.

Based on the findings from this study, it is important that outreach messages focus on reducing mental health stigma and encouraging psychological help-seeking among male SSM/V. This may involve developing a strategic communication plan that addresses elements of military culture that perpetuate mental health stigma and thwart efforts to engage in help-seeking. For example, these efforts could focus on misconceptions surrounding toughness, masculinity, and chain-of-command. These messages should be informed by the needs assessment and current literature, developed in conjunction with the Veterans Advocate, and disseminated in ways that are sensitive to the saliency of military identity.

Finally, it is recommended that university counseling centers continue to prioritize the needs of SSM/V. Targeted programming and collaborative efforts between student services and the Veterans Affairs office could positively contribute to this prioritization. Faculty and staff should be provided with effective training for working with SSM/V. In doing so, Hayden (2014) noted, "Such trainings have the potential to increase the ability of campus faculty and staff to identify distressed students, make necessary referrals, create a welcoming classroom environment, and learn how to accommodate course material to the variable schedule of student veteran needs" (p. 19). Further, university counseling centers could develop a network of offcampus providers including VA hospitals, veterans centers, and private providers to ensure that appropriate referrals are provided to SSM/V. University counseling centers could identify staff members that have an interest in working with this population and a strong working knowledge of the complex landscape of military culture. In collaboration with the Veterans Affairs office, these individuals could conduct outreach for SSM/V to promote psychological help-seeking and 
foster trust between SSM/V and counseling services. University counseling centers could also consider developing in-house programming specific for SSM/V including workshops and a military student support groups, in addition to didactic training for staff members on best practices for providing therapeutic support to SSM/V.

\section{Future Directions}

This study opens the door for future research on psychological help-seeking among SSM/V. Based on previous research, this study examined self-stigma, public-stigma, and gender as predictors of attitudes toward psychological help-seeking. Future studies could expand on this study by examining other predictors such as combat deployment. For example, existing literature has demonstrated a significant increase in self-reported mental health issues between pre-deployment and post-deployment combat veterans (Hoge et al., 2014). These issues include elevated levels of generalized anxiety, depression, post-traumatic stress disorder (PTSD), highrisk alcohol use, and suicidality (Bonar et al., 2014; Rudd, Goulding, \& Bryan, 2011). The prevalence of mental health issues among combat-exposed veterans leads the researcher to wonder whether there is a relationship between combat-exposure and attitudes toward psychological help-seeking.

Outside of gender, future research could also examine other demographic variables including academic status, military branch, age, or race/ethnicity as predictors of attitudes toward psychological help-seeking. For example, results from the current study revealed a small positive effect for age and attitudes toward psychological help-seeking where increased age was correlated with more positive attitudes toward psychological help-seeking, $r(101)=.239, p=$ .015. Further, there was a small negative effect for military status and attitudes toward psychological help-seeking such that active duty servicemembers and reservists endorsed more 
negative attitudes toward psychological help-seeking than veterans, $r(101)=-.230, p=.02$. Future literature could examine these variables more closely.

This line of research could also be advanced by utilizing other research designs, including a qualitative or mixed-methods design. While survey research offers several advantages (Heppner et al., 1999), this type of research does not provide a deep understanding of complex psychological concepts. Qualitative methodology, on the other hand, can offer robust insight into constructs that can be difficult to quantify. Therefore, it may be useful to explore psychological help-seeking through a mixed-methods design in which the data can be triangulated through a combination of quantitative and qualitative methodology. Finally, it is recommended that future studies on this topic ask participants about their psychological and psychiatric treatment history. It may be important to explore the effect, or lack thereof, of one's previous treatment experiences on their perceptions and attitudes toward psychological helpseeking.

\section{Conclusion}

The increasing number of SSM/V on today's college campuses warrants increased empirical and clinical interest about the psychological needs of military students. Previous literature on psychological help-seeking among military and college student populations has prepared the groundwork for future work on SSM/V. This study sought to expand on previous research by examining predictors of psychological help-seeking among SSM/V. Consistent with the literature, self-stigma and gender significantly predicted attitudes toward psychological helpseeking among SSM/V. The finding demonstrated that self-stigma fully mediated the significant relationship between public stigma and attitudes toward psychological help-seeking. Results also 
indicated that female SSM/V endorsed significantly more positive attitudes toward psychological help-seeking than male SSM/V.

Considering past literature on military and college student populations, these findings were expected. That said, the deeper implications of this study for administrators and clinicians on college campuses are perhaps the most important contributions of this study. The current study serves as a springboard to other psychological literature concerning the experiences of SSM/V, a student population who can bring many strengths to the classroom (Ang \& Kelley, 2016). This study demonstrated that, in fact, SSM/V experience barriers to psychological helpseeking that may prevent them from seeking services they need to be successful. This study opens the door for greater discourse, both in science and practice, about the psychological needs of SSM/V. Ultimately, this discourse could facilitate access to psychological services for SSM/V that improve their psychological wellbeing and help them to transition from military service to civilian life. 


\section{References}

Ackerman, R., DiRamio, D., \& Garza Mitchell, R. L. (2009). Transitions: Combat veterans as college students. New Directions for Student Services, 26, 5-14. https://doi.org/10.1002/ss.311

American College Health Association. (2011). 2010 National college health assessment. Washington, DC: American College Health Association. Retrieved from http://www.acha-ncha.org/docs/acha-nchaii_referencegroup_executivesummary_fall2010.pdf

American Psychological Association. (2002). Guidelines on multicultural education, training, research, practice, and organizational change for psychologists. Washington, DC: Author.

Ang, T., \& Kelley, B. (2016). 5 strengths military-connected students bring to your campus. Supporting Military-Connected Students for Success and Completion Digital Recording. Recorded podcast retrieved from https://www.academicimpressions.com/5-strengthsmilitary-connected-students-bring-to-your-campus/

Angermeyer, M.C., \& Dietrich, S. (2006). Public beliefs about and attitudes toward people with mental illness: A review of population studies. Acta Psychiatrica Scandinavica, 113(3), 163-179. https://doi.org/10.1111/j.1600-0447.2005.00699.x

Barney, L. J., Griffiths, K. M., Jorm, A. F., \& Christensen, H. (2006). Stigma about depression and its impact on help-seeking intentions. Australian and New Zealand Journal of Psychiatry, 40(1), 51-54. doi:10.1111/j.1440-1614.2006.01741.x

Baron, K.G., Lattie, E., Ho, J., \& Mohr, D.C. (2013). Interest and use of mental health and specialty behavioral medicine counseling in US primary care patients. International Society of Behavioral Medicine, 20, 69-76. https://doi.org/10.1007/s12529-011-9211-4 
Baron, R. M., \& Kenny, D. A. (1986). The moderator-mediator variable distinction in social psychological research: Conceptual, strategic, and statistical considerations. Journal of Personality and Social Psychology, 51(6), 1173-1182. http://dx.doi.org/10.1037/00223514.51.6.1173

Barry, A.E., Whiteman, S.D., Wadsworth, S.M., \& Hitt, S. (2012). The alcohol use and associated mental health problems of student servicemembers/veterans in higher education. Drugs: Education, Prevention, \& Policy, 19(5), 415-425. https://doi.org/10.3109/09687637.2011.647123

Barry, A.E., Whiteman, S.D., \& Wadsworth, S.M. (2014). Student servicemembers/veterans in higher education: A systematic review. Journal of Student Affairs Research and Practice, 51(1), 30-42. https://doi.org/10.1515/jsarp-2014-0003

Baruch, Y. (1999). Response rate in academic studies: A comparative analysis. Human Relations, 52(4), 421-438. https://doi.org/10.1177/001872679905200401

Baruch, Y., \& Holtom B.C. (2008). Survey response rate levels and trends in organizational research. Human Relations, 61(8), 1139-1160. https://doi.org/10.1177/0018726708094863

Blais, R.K., \& Renshaw, K.D. (2014). Self-stigma fully mediates that association of anticipated enacted stigma and help-seeking intentions in national guard servicemembers. Military Psychology, 26(2), 114-119. https://doi.org/10.1037/mil0000036

Bonar, E.E., Bohnert, K.M., Walters, H.M., Ganoczy, D., \& Valenstein, M. (2014). Student and nonstudent national guard servicemembers/veterans and their use of services for mental health symptoms. Journal of American College Health, 63(7), 437-446. https://doi.org/10.1080/07448481.2014.975718 
Brockington, I.F., Hall, P., Levings, J., \& Murphy, C. (1993). The community's tolerance of mentally ill. British Journal of Psychiatry, 162(1), 93-99. https://doi.org/10.1192/bjp.162.1.93

Bryan, C.J., \& Bryan, A.O. (2015). Sociodemographic correlates of suicidal thoughts and behaviors among college student servicemembers/veterans. Journal of American College Health, 63(7), 502-507. https://doi.org/10.1080/07448481.2014.939982

Bryan, C.J., Bryan, A.O., Hinkson, K., Jr., Bichrest, M., \& Ahern, D.A. (2014). Depression, posttraumatic stress disorder, and grade point average among student servicemembers and veterans. Journal of Rehabilitation Research \& Development, 51(7), 1035-1055. https://doi.org/10.1682/jrrd.2014.01.0012

Campbell, M., \& Riggs, S.A. The role of psychological symptomology and social support in the academic adjustment of previously deployed student veterans. Journal of American College Health, 63(7), 473-481. https://doi.org/10.1080/07448481.2015.1040408

Cohen, J. (1988). Statistical power analysis for the behavioral sciences (2nd ed.). Abingdon, UK: Routledge.

College Board's Annual Survey of Colleges. (2016). West Virginia University [dataset]. Retrieved from https://bigfuture.collegeboard.org/college-university-search/westvirginia-university

Cook, D.R., \& Weisberg, S. (1982). Residuals and influence in regression. New York, NY: Chapman and Hall.

Corrigan, P. W. (1998). The impact of stigma on severe mental illness. Cognitive and Behavioral Practice, 5(2), 201-222. https://doi.org/10.1016/s1077-7229(98)80006-0 
Corrigan, P. W., \& Penn, D. L. (1999). Lessons from social psychology on discrediting psychiatric stigma. American Psychologist, 54(9), 765-776. https://doi.org/10.1037//0003-066x.54.9.765

Corrigan P. W., \& Watson, A. C. (2002). The paradox of self-stigma and mental illness. Clinical Psychology: Science and Practice, 9(1), 35-53. https://doi.org/10.1093/clipsy/9.1.35

Corrigan, P. W. (2004). How stigma interferes with mental health care. American Psychologist, 59(7), 614-625. https://doi.org/10.1037/0003-066x.59.7.614

Deane, F.P., \& Chamberlain, K. (1994). Treatment fearfulness and distress as predictors of professional psychological help-seeking. British Journal of Guidance \& Counselling, 22 (2), 207-218. https://doi.org/10.1080/03069889400760211

Department of Defense. (2014). 2014 demographics: Profile of the military community. Retrieved from http://download.militaryonesource.mil/12038/MOS/Reports/2014Demographics-Report.pdf

Department of Veteran Affairs (2014). Education Program Beneficiaries. Retrieved from https://www.va.gov/vetdata/docs/QuickFacts/Education_Beneficiaries.pdf

Department of Veteran Affairs (2016). Profile of post-9/11 veterans: 2014. Retrieved from https://www.va.gov/vetdata/docs/SpecialReports/Post_911_Veterans_Profile_2014.pdf

DiRamio, D., Ackerman, R., Mitchell, R.L., \& Garza Mitchell, R. (2008). From combat to campus: Voices of student-veterans. NASPA Journal, 45(1), 73-102. https://doi.org/10.2202/1949-6605.1908

Dortch, C. (2014). The Post-9/11 Veterans Educational Assistance Act of 2008 (Post-9/11 GI Bill): Primer and Issues. Retrieved from https://fas.org/sgp/crs/misc/R42755.pdf 
Dunivin, K.O. (1997). Military culture: A paradigm shift? Retrieved from http://www.au.af.mil/au/awc/awcgate/maxwell/mp10.pdf

Eisenberg, D., Speer, N., \& Hunt, J. B. (2012). Attitudes and beliefs about treatment among college students with untreated mental health problems. Psychiatric Services, 63(7), 711713. https://doi.org/10.1176/appi.ps.201100250

Elhai, J.D., Schweinle, W., \& Anderson, S.M. (2008). Reliability and validity of the attitudes toward seeking professional psychological help scale-short form. Psychiatry Research, 159(3), 320-329. https://doi.org/10.1016/j.psychres.2007.04.020

Faul, F., Erdfelder, E., Lang, A.-G., \& Buchner, A. (2007). G*Power 3: A flexible statistical power analysis program for the social, behavioral, and biomedical sciences. Behavior Research Methods, 39(2), 175-191. https://doi.org/10.3758/bf03193146

Field, A. (2013). Discovering Statistics Using IBM SPSSS Statistics (4th ed.). London: SAGE Publications.

Fischer, E.H., \& Farina, A. (1995). Attitudes toward seeking professional psychological help: A shortened form and considerations for research. Journal of College Student Development, 36, 368-373. https://doi.org/10.1037/t05375-000

Fischer, E.H., \& Turner, J.I. (1970). Orientations to seeking professional help: Development and research utility of an attitude scale. Journal of Consulting and Clinical Psychology, 35(1), 79-90. https://doi.org/10.1037/h0020198

Fuller, J., Edwards, J., Procter, N., \& Moss, J. (2000). How definitions of mental health problems can influence help seeking in rural and remote communities. The Austrian Journal of Rural Health, 8(3), 148-153. https://doi.org/10.1046/j.1440-1584.2000.00303.x 
Griffin, K. A., \& Gilbert, C. K. (2015). Better transitions for troops: An application of Schlossberg's transition framework to analyses of barriers and institutional support structures for student veterans. Journal of Higher Education, 86(1), 71-97. https://doi.org/10.1080/00221546.2015.11777357

George, D., \& Mallery, P. (2003). SPSS for Windows step by step: A simple guide and reference (4th ed.). Boston: Allyn \& Baco.

Hayden, J.C. (2014). Serving those who served: Understanding the needs of student veterans (thesis). Retrieved from http://commons.lib.jmu.edu/edspec201019/52

Held, P., \& Owens, G.P. (2012). Stigmas and attitudes toward seeking mental health treatment in a sample of veterans and active duty servicemembers. Traumatology, 19(2), 136-143. https://doi.org/10.1177/1534765612455227

Heppner, P.P., Kivlighan, D.M., Jr., \& Wampold, B.E. (1999). Research design in counseling (2nd ed.). New York: Brooks/Cole.

Hoge, C. W., Auchterlonie, J. L., \& Milliken, C. S. (2006). Mental health problems, use of mental health services, and attrition from military service after returning from deployment to iraq or afghanistan. JAMA, 295(9), 1023. doi:10.1001/jama.295.9.1023

Hsu, J. (2010). Overview of military culture [PowerPoint slides]. Retrieved from http://sttpml.org/wp-content/uploads/2014/06/military-culture.pdf

Jakupcak, M., Blais, R. K., Grossbard, J., Garcia, H., \& Okiishi, J. (2014). "Toughness" in association with mental health symptoms among Iraq and Afghanistan war veterans seeking veterans affairs health care. Psychology of Men and Masculinity, 15(1), 100-104. https://doi.org/10.1037/a0031508 
Kelly, A.E., \& Achter, J.A. (1995). Self-concealment and attitudes toward counseling in university students. Journal of Counseling Psychology, 42(1), 40-46. https://doi.org/10.1037//0022-0167.42.1.40

Kennedy, C.H., Jones, D.E., \& Arita, A.A. (2007). Multicultural experiences of U.S. military psychologists: Current trends and training target areas. Psychological Services, 4(3), 158-167. https://doi.org/10.1037/1541-1559.4.3.158

Kline, P. (1999). The handbook of psychological testing (2nd ed.). London: Routledge.

Komiya, N., Good, G. E., \& Sherrod, N. B. (2000). Emotional openness as a predictor of college students' attitudes toward seeking psychological help. Journal of Counseling Psychology, 47(1), 138-143. https://doi.org/10.1037//0022-0167.47.1.138

Kulesza, M., Pedersen, E., Corrigan, P., \& Marshall, G. (2015). Help-Seeking Stigma and Mental Health Treatment Seeking Among Young Adult Veterans. Military Behavioral Health, 3(4), 230-239. http://doi.org/10.1080/21635781.2015.1055866

Lally, J., O'Conghaile, A., Quigley, S., Bainbridge, E., \& McDonald, C. (2013). Stigma of mental illness and help-seeking intention in university students. The Psychiatrist Online, 37(8), 253-260. https://doi.org/10.1192/pb.bp.112.041483

Link, B., Cullen, F., Struening, E., Shrout, P., \& Dohrenwend, B. (1989). A modified labeling theory approach to mental disorders: An empirical assessment. American Sociological Review, 54(3), 400-423. https://doi.org/10.2307/2095613

Livingston, W. G. (2009). Discovering the academic and social transitions of re-enrolling student veterans at one institution: A grounded theory. Retrieved from ProQuest Digital Dissertations. 
Livingston, W.G., Scott, D.A., Havice, P.A., \& Cawthon, T.W. (2012). Social camouflage: Interpreting male student veterans' behavior for residence life professionals. The Journal of College and University Student Housing, 39(1), 176-185. http://www.acuho-i.org/

Love, S.M., Levin, A., \& Park, H-S. (2015). Exploring student servicemembers/veterans social support and campus climate in the context of recovery. Social Sciences, 4, 909-924. doi:10.3390/socsci4040909

Mahalik, J.R., Locke, B.D., Ludlow, L.H., Demer, M.A., Scott, R.P., Gottfried, M. \& Freitas, G. (2003). Development of the conformity to masculine norms inventory. Psychology of Men and Masculinity. 4(1), 3-25. doi:10.1037//1524-9220.4.1.3

National Conference of State Legislatures. (2014). Veterans and college. Retrieved from http://www.ncsl.org/research/education/veterans-and-college.aspx

National Institute of Mental Health Services (2012). Results from the 2012 national survey on drug use and health: Mental health findings. Retrieved from https://www.samhsa.gov/data/sites/default/files/NSDUHmhfr2013/NSDUHmhfr2013.pdf

National Survey of Student Engagement. (2010). Major differences: Examining student engagement by field of study: Annual results 2010. Retrieved from http://nsse.indiana.edu/NSSE_2010_Results/pdf/NSSE_2010_AnnualResults.pdf

Niv, N., \& Bennett, L. (2017). Veterans' mental health in higher education settings: Services and clinician education needs. Psychiatric Services, 68(6), 636-639. https://doi.org/10.1176/appi.ps.201600065

Olsen, T., Badger, K., \& McCuddy, M.D. (October 2014). Understanding the student veterans' college experience: An exploratory study. Army Medical Department Journal, 101-108. http://www.cs.amedd.army.mil/amedd_journal.aspx 
O’Neil, J. M. (1981a). Male sex-role conflict, sexism, and masculinity: Implications for men, women, and the counseling psychologist. The Counseling Psychologist, 9, 61-80. https://doi.org/10.1177/001100008100900213

O’Neil, J. M. (1981b). Patterns of gender role conflict and strain: Sexism and fear of femininity in men's lives. Personnel and Guidance Journal, 60, 203-210. https://doi.org/10.1002/j.2164-4918.1981.tb00282.x

O’Neil, J.M. (2008). Summarizing 25 years of research on men's gender role conflict using the gender role conflict scale. The Counseling Psychologist, 36(3), 385-445. https://doi.org/10.1177/0011000008317057

O’Neil, J. M., Good, G. E., \& Holmes, S. (1995). Fifteen years of theory and research on men’s gender role conflict: New paradigms for empirical research. In R. Levant \& W. Pollack (Eds.), The new psychology of men (pp. 164-206). New York: Basic Books.

O’Neil, J. M., Helms, B.J., Gable, R.K., David, L., \& Wrightsman, L. (1986). Gender Role Conflict Scale (GRCS): College men's fears of femininity. Sex Roles, 14(5-6), 335-350. https://doi.org/10.1007/bf00287583

Ouimette, P., Vogt, D., Wade, M., Tirone, V., Greenbaum, M. A., Kimerling, R., \& Rosen, C. S. (2011). Perceived barriers to care among veterans health administration patients with posttraumatic stress disorder. Psychological Services, 8(3), 212-223. https://doi.org/10.1037/a0024360

Pattyn, E., Verhaeghe, M., Sercu, C., \& Bracke, P. (2014). Public stigma and self-stigma: Differential association with attitudes toward formal and informal help seeking. Psychiatric Services, 65(2), 232-238. https://doi.org/10.1176/appi.ps.201200561 
Pederson, E.L., \& Vogel, D.L. (2007). Men's gender role conflict and their willingness to seek counseling: Testing a mediation model on college-aged men. Journal of Counseling Psychology, 54(4), 373-384. https://doi.org/10.1037/0022-0167.54.4.373

Reetz, D.R., Barr, V., \& Krylowicz, B. (2014). The 2013 Association for University and College Counseling Center Directors Annual Survey. CO: AUCCCD.

Rickwood, D., \& Thomas, K. (2012). Conceptual measurement framework for help-seeking for mental health problems. Psychology Research and Behavior Management, 5, 173-183. https://doi.org/10.2147/prbm.s38707

Rickwood, D., Deane, F.P., Wilason, C.J., \& Ciarrochi, J. (2005). Young people’s help-seeking for mental health problems. Australian e-Journal for the Advancement of Mental Health, 4(3), 1-34. https://doi.org/10.5172/jamh.4.3.218

Robinson Kurpius, S. E., \& Lucart, A. L. (2000). Military and civilian undergraduates: Attitudes toward women, masculinity, and authoritarianism. Sex Roles, 43(3-4), 255-265. http://www.springer.com/psychology/journal/11199

Romero, D.H., Riggs, S.A., \& Ruggero, C. (2015). Coping, family social support, and psychological symptoms among student veterans. Journal of Counseling Psychology, 62(2), 242-252. https://doi.org/10.1037/cou0000061

Rudd, M. D., Goulding, J., \& Bryan, C. J. (2011). Student veterans: A national survey exploring psychological symptoms and suicide risk. Professional Psychology: Research and Practice, 45(5), 354-360. https://doi.org/10.1037/a0025164

Rumann, C. B., \& Hamrick, F. A. (2010). Student veterans in transition: Re-enrolling after war zone deployments. Journal of Higher Education, 81(4), 431-458.

https://doi.org/10.1080/00221546.2010.11779060 
Schonfeld, L., Braue, L.A., Stire, S., Gum, A.M., Cross, B.L., Brown, L.M. (2015). Behavioral health and adjustment to college life for student service members/veterans, Journal of American College Health, 63(7), 428-436, doi: 10.1080/07448481.2014.963106

Shaffer, P.A., Vogel, D.L., \& Wei, M. (2006). The mediating roles of anticipated risks, anticipated benefits, and attitudes on the decision to seek professional help: An attachment perspective. Journal of Counseling Psychology, 53(4), 442-452. https://doi.org/10.1037/0022-0167.53.4.442

Substance Abuse and Mental Health Services Administration. (2013). Results from the 2012 national survey on drug use and health: Mental health findings. Retrieved from http://www.samhsa.gov/data/NSDUH/2k12MH_FindingsandDetTables/2K12MHF/NSD UHmhfr2012.htm\#sec2-4

Thompson, E. H., \& Pleck, J. H. (1986). The structure of male role norms. American Behavioral Scientist, 29(5), 531-543. https://doi.org/10.1177/000276486029005003

Topkaya, N. (2014). Gender, self-stigma, and public stigma in predicting attitudes toward psychological help-seeking. Educational Sciences: Theory, \& Practice, 14(2), 480-487. https://doi.org/10.12738/estp.2014.2.1799

Topkaya, N. (2015). Factors influencing psychological help seeking in adults: A qualitative study. Educational Sciences: Theory, \& Practice, 15(1), 21-31. https://doi.org/10.12738/estp.2015.1.2094

Vogel, D.L., Wade, N.G., \& Haake, S. (2006). Measuring the self-stigma associated with seeking psychological help. Journal of Counseling Psychology 53(3), 325-337. https://doi.org/10.1037/0022-0167.53.3.325 
Vogel, D.L., \& Wei, M. (2005). Adult attachment and help-seeking intent: The mediating roles of psychological distress and perceived social support. Journal of Counseling Psychology, 52(3), 347-357. https://doi.org/10.1037/0022-0167.52.3.347

Vogel, D.L., Wester, S.R., Wei, M., \& Boysen, G.A. (2005). The role of outcome expectations and attitudes on decisions to seek professional help. Journal of Counseling Psychology, 52(4), 459-470. https://doi.org/10.1037/0022-0167.52.4.459

Vogel, D.L., Heimerdinger-Edwards, S.R., Hammer, J.H., \& Hubbard, A. (2011). “Boys don’t cry": Examination of the links between endorsement of masculine norms, self-stigma, and help-seeking attitudes for men from diverse backgrounds. Journal of Counseling Psychology, 58(3), 368-382. https://doi.org/10.1037/a0023688

Vogel, D.L., Shechtman, Z., \& Wade, N.G. (2010). The role of public and self-stigma in predicting attitudes toward group counseling. The Counseling Psychologist, 38(7), 904922. https://doi.org/10.1177/0011000010368297

Vogel, D.L., Wade, N.G., \& Hackler, A.H. (2008). Emotional expression and the decision to seek therapy: The mediating roles of the anticipated benefits and risks. Journal of Social and Clinical Psychology, 27(3), 254-278. https://doi.org/10.1521/jscp.2008.27.3.254

Vogel, D.L., Wester, S.R., Hammer, J.H., \& Downing-Matibag, T.M. (2014). Referring men to seek help: The influence of gender role conflict and stigma. Psychology of Men \& Masculinity, 15(1), 60-67. https://doi.org/10.1037/a0031761

Wampold, B.E. (2001). The great psychotherapy debate: Models, methods, and findings. Mahwah, NJ: Erlbaum.

Weiner, B. (1985). An attributional theory of achievement motivation and emotion. Psychological Review, 92(4), 548-573. doi:10.1037/0033-295X.92.4.548 


\section{APPENDIX A \\ INFORMATIONAL COVER LETTER}

April 19, 2017

Dear Participant,

This letter is to request your participation in a research study on help-seeking among student servicemembers and veterans. This project is acknowledged by the Institutional Review Board (IRB) at West Virginia University (WVU) and is being conducted by Melissa Foster in the Department of Counseling, Rehabilitation Counseling, and Counseling Psychology under the guidance of Dr. Monica Leppma, Assistant Professor. Your participation in this project is highly valued and will take approximately 10 minutes via an online survey. You can access the survey at http://wvu.qualtrics.com/jfe/form/SV_23Pm8aKQ7ZBBIax.

Your involvement in this project will be kept as confidential as legally possible as no identifying information will be collected. While you will have the option of providing an email address to enter a raffle, this information will be kept separate from your individual responses. In order to participate, you must be a student service member or veteran currently enrolled in a degreeseeking program and at least 18 years of age or older. Your participation is completely voluntary. You may skip any question that you do not wish to answer and you may discontinue participation at any time. Your class standing and military status will not be affected by your refusal to participate or your withdrawal from the study.

Your participation is very much appreciated. Please do not hesitate to contact Melissa Foster (mnfoster@mix.wvu.edu) or Dr. Leppma (monica.leppma@mail.wvu.edu) should you have any questions or concerns.

Thank you!

Sincerely,

Melissa Foster, MEd.

Counseling Psychology Doctoral Candidate

Department of Counseling, Rehabilitation Counseling, and Counseling Psychology

West Virginia University | Morgantown, WV 


\section{APPENDIX B \\ INFORMED CONSENT}

\section{Only Minimal Risk Consent Information Form (without HIPAA)}

Principal Investigator: Monica Leppma, $\mathrm{PhD}$.

Department: Counseling, Rehabilitation Counseling \& Counseling Psychology

Protocol Number: 1611350967

Study Title: From Service Member to Student: Predictors of Psychological Help-Seeking Attitudes in Student Servicemembers and Veterans

Co-Investigator: Melissa Foster, MEd.

\section{Contact Persons}

Research at West Virginia University (WVU) is carried out under the oversight of the Institutional Review Board (IRB). This study has been reviewed and approved by the IRB at WVU. In the event that you experience any side effects or injury related to this research, you should contact Monica Leppma, PhD. during business hours at (304) 293-0540, after hours at (772) 532-6844), or at monica.leppma @ mail.wvu.edu. If you have any questions, concerns, or complaints about this research, please contact Dr. Leppma. Alternatively, you can contact Melissa Foster, MEd. at mnfoster@mix.wvu.edu or (757) 647-9037. For information regarding your rights as a research subject, to discuss problems, concerns, or suggestions related to the research, to obtain information, or to offer input about this research, contact the Office of Research Integrity \& Compliance at WVU at (304) 293-7073.

\section{Introduction}

You have been asked to participate in this research study, which has been explained to you by Dr. Leppma and Melissa Foster via e-mail. This study is being conducted by Dr. Leppma and Melissa Foster in the Department of Counseling, Rehabilitation Counseling, and Counseling Psychology at WVU. You were invited to participate in this study because you have been identified as a student service member or veteran.

\section{Purpose of the Study}

The purpose of this study is to better understand predictors of psychological help-seeking attitudes among student servicemembers and veterans.

\section{Description of Procedures}

This study involves completing a voluntary and anonymous online survey. Participation will take approximately 10 minutes. You do not have to answer all the questions and you have the right to terminate your participation at any time.

\section{Discomforts}

There are minimal risks associated with this study.

\section{Benefits}

There are no specific benefits in terms of your participation in this study. However, the aims of 
the overall study may benefit student service member and veterans, in addition to future research surrounding this population.

\section{Financial Considerations}

There are no special fees for participating in this study. You will not be paid for participating. At the end of the study, participants will have the opportunity to submit an email address to be entered into a random drawing (randompicker.com) to win one of six \$25 Amazon electronic gift certificates. Within a month of closing the survey, six email addresses will be drawn at random and the winners will be emailed a link to their electronic gift certificate. Email addresses for drawing participants will not be associated with individual responses and will be stored in a separate password-protected document.

\section{Confidentiality}

Any information about you that is obtained as a result of your participation in this research will be kept as confidential as legally possible. Your research records and test results, just like hospital records, may be subpoenaed by court order or may be inspected by the study sponsor or federal regulatory authorities (including the FDA if applicable) without your additional consent. In addition, there are certain instances where the researcher is legally required to give information to the appropriate authorities. These would include mandatory reporting of infectious diseases, mandatory reporting of information about behavior that is imminently dangerous to a child or to others, such as suicide, child abuse, etc. In any publications that result from this research, neither your name, email address, or any identifiable information will be published without your consent.

\section{Voluntary Participation}

Participation in this study is voluntary. You are free to withdraw your consent to participate in this study at any time. In the event that new information becomes available that may affect your willingness to participate in this study, this information will be provided to you so that you can make an informed decision about whether or not to continue with participation. You have been given the opportunity to ask questions about the research, and you have received answers concerning areas you did not understand.

I voluntarily agree to participate in this study. 


\section{APPENDIX C \\ DEMOGRPAHICS DATA FORM}

Please complete the following information.

\begin{tabular}{|c|c|c|}
\hline TEXT & ANSWERS & TYPE \\
\hline Gender: & $\begin{array}{ll}\circ & \text { Male } \\
\circ & \text { Female } \\
\circ & \text { Self-Identify: } \\
\end{array}$ & Multiple Choice \\
\hline Age: & $\longrightarrow$ & Text Entry \\
\hline What is your race/ethnicity? & $\begin{array}{ll}\circ & \text { American Indian or Alaska Native } \\
\circ & \text { Asian American/Asian } \\
\circ & \text { Black/African American } \\
\circ & \text { Hispanic/Latino/a } \\
\circ & \text { Multiracial } \\
\circ & \text { Native Hawaiian/Pacific Islander } \\
\circ & \text { White/Caucasian } \\
\circ & \text { Self-Identify: } \\
\end{array}$ & Multiple Choice \\
\hline Current academic status: & $\begin{array}{ll}\circ & \text { Freshman/First-Year } \\
\circ & \text { Sophomore } \\
\circ & \text { Junior } \\
\circ & \text { Senior } \\
\circ & \text { Graduate/professional degree seeking } \\
& \text { student } \\
\circ & \text { Non-degree seeking student } \\
\circ & \text { Other: }\end{array}$ & Multiple Choice \\
\hline Current type of program: & $\begin{array}{ll}\circ & \text { Technical certification } \\
\circ & \text { Associate's degree } \\
\circ & \text { Bachelor's degree } \\
\circ & \text { Graduate/professional degree } \\
\circ & \text { Other: }\end{array}$ & Multiple Choice \\
\hline Institution name: & $\begin{array}{ll} & \text { West Virginia University } \\
\circ & \text { Other: }\end{array}$ & Multiple Choice \\
\hline
\end{tabular}




\begin{tabular}{|c|c|c|}
\hline Military branch: & $\begin{array}{ll}\circ & \text { Army } \\
\circ & \text { Navy } \\
\circ & \text { Air Force } \\
\circ & \text { Marine Corps } \\
\circ & \text { Coast Guard } \\
\circ & \text { Commissioned Officer of the PHS } \\
\circ & \text { Commissioned Officer of the NOAA } \\
\circ & \text { Other: }\end{array}$ & Multiple Choice \\
\hline Current military status: & $\begin{array}{ll}\circ & \text { Active-duty } \\
\circ & \text { National Guard/Reserve } \\
\circ & \text { Veteran } \\
\circ & \text { Other: }\end{array}$ & Multiple Choice \\
\hline $\begin{array}{l}\text { Number of years of military } \\
\text { service: }\end{array}$ & & Text Entry \\
\hline $\begin{array}{l}\text { Have you been previously } \\
\text { deployed to a combat/armed } \\
\text { conflict zone? }\end{array}$ & $\begin{array}{ll}\circ & \text { Yes } \\
\circ & \text { No }\end{array}$ & Multiple Choice \\
\hline $\begin{array}{l}\text { If yes, how many times have you } \\
\text { been deployed to a combat/armed } \\
\text { conflict zone? }\end{array}$ & & Text Entry \\
\hline $\begin{array}{l}\text { If yes, where have you been } \\
\text { deployed (please select all that } \\
\text { apply)? }\end{array}$ & $\begin{array}{ll}\circ & \text { Iraq } \\
\circ & \text { Afghanistan } \\
\circ & \text { Other: }\end{array}$ & Multiple Choice \\
\hline
\end{tabular}




\section{APPENDIX D \\ SELF-STIGMA OF SEEKING HELP SCALE}

Please rate your level of agreement with the statements below.

I would feel inadequate if I went to a therapist for psychological help.

$\begin{array}{ccccc}\begin{array}{c}\text { Strongly } \\ \text { Disagree }\end{array} & \text { Disagree } & \begin{array}{c}\text { Agree and } \\ \text { Disagree } \\ \text { Equally }\end{array} & \text { Agree } & \text { Strongly Agree } \\ 1 & 2 & 3 & 4 & 5\end{array}$

My self-confidence would NOT be threatened if I sought professional help.

$\begin{array}{ccccc}\begin{array}{c}\text { Strongly } \\ \text { Disagree }\end{array} & \text { Disagree } & \begin{array}{c}\text { Agree and } \\ \text { Disagree } \\ \text { Equally }\end{array} & \text { Agree } & \text { Strongly Agree } \\ 1 & 2 & 3 & 4 & 5\end{array}$

Seeking psychological help would make me feel less intelligent.

$\begin{array}{ccccc}\begin{array}{c}\text { Strongly } \\ \text { Disagree }\end{array} & \text { Disagree } & \begin{array}{c}\text { Agree and } \\ \text { Disagree } \\ \text { Equally }\end{array} & \text { Agree } & \text { Strongly Agree } \\ 1 & 2 & 3 & 4 & 5\end{array}$

My self-esteem would increase if I talked to a therapist.

$\begin{array}{ccccc}\begin{array}{c}\text { Strongly } \\ \text { Disagree }\end{array} & \text { Disagree } & \begin{array}{c}\text { Agree and } \\ \text { Disagree } \\ \text { Equally }\end{array} & \text { Agree } & \text { Strongly Agree } \\ 1 & 2 & 3 & 4 & 5\end{array}$

My view of myself would not change just because I made the choice to see a therapist.

Strongly
Disagree

1
Disagree

2
Agree and

Disagree Equally
Agree

4

Strongly Agree

$$
3
$$

It would make me feel inferior to ask a therapist for help.

$$
\begin{aligned}
& \text { Strongly } \\
& \text { Disagree }
\end{aligned}
$$

1
Disagree

2
Agree and

Disagree

Equally
Agree Strongly Agree

4
5 
I would feel okay about myself if I made the choice to seek professional help.

$\begin{array}{ccccc}\begin{array}{c}\text { Strongly } \\ \text { Disagree }\end{array} & \text { Disagree } & \begin{array}{c}\text { Agree and } \\ \text { Disagree } \\ \text { Equally }\end{array} & \text { Agree } & \text { Strongly Agree } \\ 1 & 2 & 3 & 4 & 5\end{array}$

If I went to a therapist, I would be less satisfied with myself.

$\begin{array}{ccccc}\text { Strongly } & \text { Disagree } & \begin{array}{c}\text { Agree and } \\ \text { Disagree } \\ \text { Equally }\end{array} & \text { Agree } & \text { Strongly Agree } \\ 1 & 2 & 3 & 4 & 5\end{array}$

My self-confidence would remain the same if I sought help for a problem I could not solve.

$\begin{array}{ccccc}\begin{array}{c}\text { Strongly } \\ \text { Disagree }\end{array} & \text { Disagree } & \begin{array}{c}\text { Agree and } \\ \text { Disagree } \\ \text { Equally }\end{array} & \text { Agree } & \text { Strongly Agree } \\ 1 & 2 & 3 & 4 & 5\end{array}$

I would feel worse about myself if I could not solve my own problems

Strongly
Disagree

1
Disagree

2
Agree and

Disagree

Equally

3
Agree Strongly Agree

4
5 


\section{APPENDIX E \\ STIGMA SCALE FOR RECEIVING PSYCHOLOGICAL HELP}

Please rate your level of agreement with the statements below.

Seeing a psychologist for emotional or interpersonal problems carries social stigma.

Strongly

Disagree

0
Disagree

1
Agree Strongly Agree

2
3

It is a sign of personal weakness or inadequacy to see a psychologist for emotional or interpersonal problems

$\begin{array}{cccc}\text { Strongly } & \text { Disagree } & \text { Agree } & \text { Strongly Agree } \\ \text { Disagree } & 1 & 2 & 3 \\ 0 & & \end{array}$

People will see a person in a less favorable way if they come to know that he/she has seen a psychologist.

Strongly

Disagree

0
Disagree

1
Agree

2
Strongly Agree

3

It is advisable for a person to hide from people that he/she has seen a psychologist.

Strongly

Disagree

0
Disagree

1
Agree

2
Strongly Agree

3

People tend to like less those who are receiving professional psychological help.

Strongly

Disagree

0
Disagree

1
Agree

2
Strongly Agree

3 


\section{APPENDIX F \\ ATTITUDES TOWARD SEEKING PROFESSIONAL PSYCHOLOGICAL HELP SCALE - SHORT FORM}

Please rate your level of agreement with the statements below.

If I believed I was having a mental breakdown, my first inclination would be to get professional attention.

$\begin{array}{cccc}\text { Disagree } & \text { Partly } & \text { Partly Agree } & \text { Agree } \\ 0 & \text { Disagree } & 2 & 3\end{array}$

The idea of talking about problems with a psychologist strikes me as a poor way to get rid of emotional conflicts.

$\begin{array}{cccc}\text { Disagree } & \text { Partly } & \text { Partly Agree } & \text { Agree } \\ 0 & \text { Disagree } & 2 & 3\end{array}$

If I were experiencing a serious emotional crisis at this point in my life, I would be confident that I could find relief in psychotherapy.

$\begin{array}{cccc}\text { Disagree } & \text { Partly } & \text { Partly Agree } & \text { Agree } \\ 0 & \text { Disagree } & 2 & 3\end{array}$

There is something admirable in the attitude of a person who is willing to cope with his or her conflicts and fears without resorting to professional help.

$\begin{array}{cccc}\text { Disagree } & \text { Partly } & \text { Partly Agree } & \text { Agree } \\ 0 & \text { Disagree } & 2 & 3\end{array}$

I would want to get psychological help if I were worried or upset for a long period of time.

$\begin{array}{cccc}\text { Disagree } & \text { Partly } & \text { Partly Agree } & \text { Agree } \\ 0 & \text { Disagree } & 2 & 3\end{array}$

I might want to have psychological counseling in the future.
Disagree
Partly
0
Disagree
Partly Agree
Agree
2 
A person with an emotional problem is not likely to solve it alone; he or she is likely to solve it with professional help.

$\begin{array}{cccc}\text { Disagree } & \text { Partly } & \text { Partly Agree } & \text { Agree } \\ 0 & \text { Disagree } & 2 & 3\end{array}$

Considering the time and expense involved in psychotherapy, it would have doubtful value for a person like me.

$\begin{array}{cccc}\text { Disagree } & \text { Partly } & \text { Partly Agree } & \text { Agree } \\ 0 & \text { Disagree } & 2 & 3\end{array}$

A person should work out his or her own problems; getting psychological counseling would be a last resort.

$\begin{array}{cccc}\text { Disagree } & \text { Partly } & \text { Partly Agree } & \text { Agree } \\ 0 & \text { Disagree } & 2 & 3\end{array}$

Personal and emotional troubles, like many things, tend to work out by themselves.

$\begin{array}{cccc}\text { Disagree } & \text { Partly } & \text { Partly Agree } & \text { Agree } \\ 0 & \text { Disagree } & 2 & 3\end{array}$




\section{APPENDIX G \\ CLOSING STATEMENT AND RESOURCES}

Thank you for your participation!

Veterans Crisis Line

https://www.veteranscrisisline.net/

1-800-273-8255 (Select “1”)

U.S. Department of Veterans Affairs

http://www.mentalhealth.va.gov/index.asp

The Real Warriors Campaign

http://www.realwarriors.net/

National Suicide Prevention Lifeline

http://suicidepreventionlifeline.org/\#

1-800-273-8255

Carruth Center for Psychological and Psychiatric Services | West Virginia University http://well.wvu.edu/ccpps 